\title{
The Requirements of Digital Transformation and Its Economic Applications To Achieve a Competitive Advantage in Major Sports Clubs In the Arab Republic of Egypt
}

\section{Assistant Prof. Dr. / Reham Amin Hamza Shehab}

Assistant Professor, Department of Sports Administration, Faculty of Physical Education for Girls, Alexandria University.

\section{Research Problem and Importance:}

Under accelerated developments of information and communication technology, many administrative directions emerged towards adopting digital transformation.

Tomas M. Siebel (2019), George Westernman (2014) defines digital transformation as a phenomenon arising from using information and communication technologies and new digital techniques synchronically to produce a lot of information to use in decision making and strategic planning. (59: 26), (47: 32)

The researcher believes that digital transformation he researcher believes that the digital transformation is changing the structure of the institution and building its strategy on digital technology and the technical capabilities it possesses to achieve value for its customers.

UNESCO (2018) and Sara Grand (2017) defines digital skills as along chain of skills of using digital appliances, communications and works applications to reach and administrate information and establishing a digital content. (41: 2), (58: 5).

Communication International Federation (2018) indicates that digital skills are subdivided to 3 levels: basic, average and advanced skills. (23: 18)

Giacomo and Antonio (2018) and Ovidiu, Peter (2013) indicate that things internet links infinite number of things via a network with availing the relevant technical support. (48: 34), (56: 167).

Bruce Sinclair (2017) indicates that computers ability to knowledge by using things internetcollected data enables us to follow up and count things. (45: 16)

Kevin L. Jackson and Scott (2018) define cloud computerization the set of computerized sources and systems on demand via internet able to avail some of integrated computer services. (54: 12)

Financial Crises of (2008) affected the confidence between governments and companies, also weak and unsafe information technologies and users' increasing fear from lack of privacy and safety resulted in the so called blue kitchen technique.

(44: 13).

David, Christophe (2019) defines blocks chain as distributing data to a great number of points spread on internet which is computers entrusted with verifying true data and operations (46: 23).

Book of Jared Tate, Andrew Knapp (2019) mentions that blocks chain consists of 4 main elements represented in: block, information. Margin and time print. (50:27)

Joel Gurin (2014) and Rob Kitchen (2014) define open data as publishing digital data on internet in an automatic legible format. (52:18), (57:17)

Julian Singh (2017) indicates that for data to be open many levels shall be considered: legal opening, technical opening and commercial opening. (53: 44).

Beshir Arnous (2007) and Srour Ali Srour (2005) defines artificial intelligence as a part of accounting science looking for developed programming methods for carrying out works and 
conclusions similar to methods alluding intelligence to human. (10: 9), (39: 26)

Mohit Sharma (2018), Khalil Abo Koura (2014) define systems of automating robot processes as non-intertwined applications requiring no technical integration with other system. (55: 11), (37: 19)

Ramadan Elmarouf (2011), Abdelsabour Elmasry (2011) and Hind Hamid (2010) define ecommerce as all business made electronically via internet. (34:34), (2: 24), (21:17)

Jim Work George Brand (2020) mentions that applying ecommerce shall depend on three factors: "E. markets, E. data exchange and internet". (51:57)

As Gil Gildner, Anya Gildner (2019) and Youssef Abo Fara (2007) define E. marketing as some efforts exerted by the company to inform purchasers of its products and services and communicating with them via internet. (49:14), (42:27)

Egypt adopter a serious direction to be a digital community as a basis of achieving integrated and permanent development, at the first step of transferring to digital economy in Egypt, the first Egyptian communication satellite, Tiba, was developed (1). (60), (68), (61: 2), (62:1).

In the sports field, there are many new attempts to attend digital transfer, as minister of youth and sports, in coordination with officials of Microsoft Egypt looked into binary cooperation in the field of digital transformation. (63:1)

Also El Ahly Club adopted the digital transformation strategy incorporated under its future plan to transfer all services provided to members electronically, also Heliopolis Club and Kuwait National Bank - Egypt signed a cooperation protocol to support and activate digital transformation system, also Smouha Club declared a protocol of cooperation with Egypt telecom to develop the internet infra-structure.

(64: 2), (65:1), (66:1), (67:2).

The researcher concluded a Pilot Study for some supreme management officials at clubs whose number reached 6 subjects to identify the status quo and procedures taken by the supreme management to make development needed for applying digital transformation. The interview results proved the supreme management's awareness of the importance of adopting digital transformation strategies.

Also the researcher concludes a second pilot study for a sample of sports clubs' employees, whose number reached (11) subjects to identify the fact of digital transformation inside sports clubs, as the results proved establishing an interactive website, the main club's data bases are being updated, the club's infrastructure is being developed and protocols of cooperating with banks are being signed to support digital transformation projects.

Also through looking into the previous studies results such as results of Nawal Abdalla (2019) (30), Hanin Abdelasalam(2019) (18), Faleh Abbas (2019) (14), Tawil Osama (2017) (40), Dina Mohamed Adel (2016) (12), digital transformation contributes to providing high quality digital governmental services, good planning for transfer to e administration in sports department is lacking.

As study of Omar Saleh (2018) (33) and Khloud Bent Salem (2019) (24) recommends that there should be an infrastructure to enable profiting from E. government services and availing human resources to develop the same and financial resources to avail systems required for concluding digital transformation.

Through the researcher open personal interviews, the survey study and the results and recommendations of previous studies, the researcher concluded the importance of activating digital transformation system in sports clubs and adopting modern communication systems to transfer all services provided to members electronically in order to achieve the satisfaction of the 
beneficiaries, and to support the competitiveness of sports clubs. Researcher to conduct the current research and its title:

This made the researcher carry out this research titled:

"The Requirements of Digital Transformation and Its Economic Applications to Achieve a Competitive Advantage in Major Sports Clubs in the Arab Republic of Egypt"

The Research Objective:

The research aims at identifying The Requirements of Digital Transformation and Its Economic Applications to Achieve a Competitive Advantage in Major Sports Clubs in the Arab Republic of Egypt via identifying:

- Digital transformation requirements: "supreme management support, digital skills, digital techniques"

- Digital transformation economic applications: "E. commerce, E. marketing"

The Research Procedures:

The research method:

The survey descriptive method was used as suitable for the research nature.

The research community:

The research community consisted of (9) great clubs from Cairo and Alexandria.

The research sample:

Table (1) Numerical Description of the Research Community Groups Subjects and the Pilot and the Main Research Sample

\begin{tabular}{|c|c|c|c|c|c|}
\hline \multirow{2}{*}{ Administrative levels } & \multirow{2}{*}{$\begin{array}{l}\text { Total research } \\
\text { community }\end{array}$} & \multirow{2}{*}{$\begin{array}{c}\text { Sample of } \\
\text { codifying } \\
\text { questionnaire } \\
\text { form }\end{array}$} & \multirow{2}{*}{ Removed forms } & \multicolumn{2}{|c|}{ Main Search Sample } \\
\hline & & & & Number & Percentages \\
\hline Supreme management : clubs boards' members & 99 & 11 & 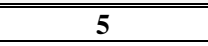 & 83 & $83.83 \%$ \\
\hline $\begin{array}{l}\text { Middle management: executive managers and } \\
\text { sports activity mangers }\end{array}$ & 25 & 6 & 3 & 18 & $64 \%$ \\
\hline Executive management: sports specialists & 123 & 21 & 13 & 89 & $72.35 \%$ \\
\hline Total & 247 & 38 & 21 & 188 & \multirow{2}{*}{$76.113 \%$} \\
\hline Percentage & $100 \%$ & $15.38 \%$ & $8.502 \%$ & $76.113 \%$ & \\
\hline
\end{tabular}

Data Collection Tools:

Questionnaire form designed by the researcher

The researcher followed the following steps to design the questionnaire form: - Results of the interview, the first and second exploratory study. - Access to the scientific references and the results of previous studies. Through this, the researcher was able to develop the factors and dimensions of the questionnaire, as it included two main factors, first factor of (3) dimensions, and second factor of (2) dimensions.

Questionnaire form scientific coefficients:

Calculating validity questionnaire form scientific coefficient:

Table (2) Correlation Coefficient between Questionnaire Dimensions and Factors $(\mathrm{N}=38)$

\begin{tabular}{|c|c|c|c|c|c|c|}
\hline \multirow{2}{*}{\multicolumn{2}{|c|}{ Factors and dimensions }} & \multicolumn{4}{|c|}{ First factor } & \multirow{3}{*}{$\begin{array}{c}\begin{array}{c}\text { Questionnaire } \\
\text { total degree }\end{array} \\
\mathbf{0 . 8 3 9} \\
\end{array}$} \\
\hline & & \multirow{2}{*}{ First factor } & \multirow{2}{*}{$\begin{array}{c}\begin{array}{c}\text { Second } \\
\text { factor }\end{array} \\
\mathbf{0 . 5 8 5} \\
\end{array}$} & \multirow{2}{*}{$\begin{array}{r}\begin{array}{r}\text { Third } \\
\text { factor }\end{array} \\
\mathbf{0 . 7 8 5} \\
\end{array}$} & \multirow{2}{*}{$\begin{array}{c}\begin{array}{c}\text { Total factors } \\
\text { degree }\end{array} \\
\mathbf{0 . 7 4 5} \\
\end{array}$} & \\
\hline First & First dimension & & & & & \\
\hline factor & Second dimension & & & 0.617 & $\mathbf{0 . 7 3 7}$ & 0.877 \\
\hline
\end{tabular}




\begin{tabular}{|c|c|c|c|c|c|}
\hline & Third dimension & & & \multirow[t]{2}{*}{0.759} & \multirow{2}{*}{$\begin{array}{l}0.851 \\
0.845 \\
\end{array}$} \\
\hline & Total degree & & & & \\
\hline \multirow{2}{*}{\multicolumn{2}{|c|}{ Factors and dimensions }} & \multicolumn{3}{|c|}{ Second factor } & Total \\
\hline & & $\begin{array}{c}\text { First } \\
\text { dimension }\end{array}$ & $\begin{array}{c}\text { Second } \\
\text { dimension }\end{array}$ & Total factors degree & $\begin{array}{c}\text { questionnaire } \\
\text { degree }\end{array}$ \\
\hline \multirow{3}{*}{$\begin{array}{l}\text { Second } \\
\text { factor }\end{array}$} & First dimension & & 0.791 & 0.534 & 0.749 \\
\hline & Second dimension & & & 0.609 & $\mathbf{0 . 8 3 0}$ \\
\hline & Total degree & & & & 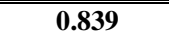 \\
\hline
\end{tabular}

Rg value $(0,05.36)=0,304$

Table (2) clarifies a statistical significant correlation at significant level (0.05) between the degree of every factor and the whole degree to prove questionnaire internal consistency.

Calculating questionnaire reliability coefficient:

Table (3) Questionnaire Reliability by Split Half and Cronbach's Alpha

\begin{tabular}{|c|c|c|c|}
\hline \multirow[b]{2}{*}{ Factors and Dimensions } & \multicolumn{2}{|c|}{ Split half } & \multirow[b]{2}{*}{ Cronbach's Alpha } \\
\hline & $\begin{array}{l}\text { Spearman } \\
\text { Brown }\end{array}$ & Guttman & \\
\hline First dimension: supreme management support & 0.716 & 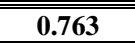 & 0.776 \\
\hline Second dimension: digital skills & 0.789 & 0.817 & 0.749 \\
\hline Third dimensions: digital technique & 0.745 & 0.736 & $\mathbf{0 . 7 0 5}$ \\
\hline $\begin{array}{l}\text { First factor: requirements of digital transformation to achieve } \\
\text { a competitive advantage at sports clubs }\end{array}$ & 0.821 & 0.755 & 0.781 \\
\hline First dimension: E. commerce & $\mathbf{0 . 7 1 5}$ & 0.873 & $\mathbf{0 . 7 7 5}$ \\
\hline Second dimension: E. marketing & $\mathbf{0 . 7 6 3}$ & 0.851 & $\mathbf{0 . 7 2 3}$ \\
\hline $\begin{array}{c}\text { Second factor: economic applications of digital transformation } \\
\text { to achieve a competitive advantage at sports clubs }\end{array}$ & 0.746 & 0.757 & 0.806 \\
\hline Total questionnaire degree & 0.721 & 0.918 & 0.881 \\
\hline
\end{tabular}

From table (3) it is clear that reliability coefficient by half split ranged between (0.715) and (0.918) and Cronbach's Alpha reliability coefficient ranged between (0.705) and (0.881) proving that the researched questionnaire has a high reliability coefficient.

Application of the questionnaire form:

The questionnaire form was applied in its final form to the basic research sample, which amounted to (188) single, during the period from (6/12/2020) to $(18 / 8 / 2020)$.

Statistical treatments:

Repetitions, percentage, spearman's rank correlation coefficient, split half for Spearman Brown and Guttman, Cronbach's Alpha reliability coefficient, outweighed percentage, arithmetic mean out weighted by weights, chi square for independent samples (cross tabulation), chi square for one sample, (Chi Square Goodness of Fit Test).

Discussing results:

Table (4) First Factor Results: Digital Transformation Requirements to Achieve the Best Competitive Advantage at Clubs (First Dimension: Supreme Management Support)

\begin{tabular}{|c|c|c|c|c|c|c|c|c|c|}
\hline $\mathbf{S}$ & Statement content & Departments & Agreeing & $\begin{array}{c}\text { To } \\
\text { a certain } \\
\text { degree }\end{array}$ & Disagreeing & $\begin{array}{c}\text { Out } \\
\text { weighted } \\
\text { percentage }\end{array}$ & Average & Attitude & Chi square \\
\hline$\overline{11}$ & & & supreme $\mathbf{m}$ & 1agement $\mathrm{s}$ & pports digital & Insformation & & & \\
\hline \multirow{4}{*}{$1 / 1$} & \multirow{4}{*}{$\begin{array}{l}\text { Looking into new } \\
\text { techniques }\end{array}$} & $\begin{array}{c}\text { Supreme } \\
\text { management }=83\end{array}$ & 66 & 9 & 8 & 89.96 & 2.70 & agreeing & \multirow{3}{*}{7.49} \\
\hline & & $\begin{array}{c}\text { Middle } \\
\text { management }=16\end{array}$ & 9 & 2 & 5 & 75.00 & 2.25 & $\begin{array}{c}\text { to a certain } \\
\text { degree }\end{array}$ & \\
\hline & & $\begin{array}{c}\text { Executive } \\
\text { management= } 89\end{array}$ & 61 & 8 & 20 & 82.02 & 2.46 & agreeing & \\
\hline & & All $=188$ & 136 & 19 & 33 & 84.93 & 2.55 & agreeing & 130.29 \\
\hline $1 / 2$ & Readiness for bearing & $\begin{array}{c}\text { Supreme } \\
\text { management }=83\end{array}$ & 62 & 13 & 8 & 88.35 & 2.65 & agreeing & $15.15 *$ \\
\hline
\end{tabular}




\begin{tabular}{|c|c|c|c|c|c|c|c|c|c|}
\hline $\mathbf{S}$ & Statement content & Departments & Agreeing & $\begin{array}{c}\text { To } \\
\text { a certain } \\
\text { degree }\end{array}$ & Disagreeing & $\begin{array}{c}\text { Out } \\
\text { weighted } \\
\text { percentage }\end{array}$ & Average & Attitude & Chi square \\
\hline & $\begin{array}{l}\text { digital transformation } \\
\text { application costs }\end{array}$ & $\begin{array}{c}\text { Middle } \\
\text { management }=16\end{array}$ & 8 & 2 & 6 & 70.83 & 2.13 & $\begin{array}{l}\text { to a certain } \\
\text { degree }\end{array}$ & \\
\hline & & $\begin{array}{c}\text { Executive } \\
\text { management }=89\end{array}$ & 54 & 7 & 28 & 76.40 & 2.29 & $\begin{array}{l}\text { to a certain } \\
\text { degree }\end{array}$ & \\
\hline & & All $=188$ & 124 & 22 & 42 & 81.21 & 2.44 & agreeing & 93.23 \\
\hline \multirow{4}{*}{$1 / 3$} & \multirow{4}{*}{$\begin{array}{l}\text { Training employees and } \\
\text { providing them with all } \\
\text { skills neede for } \\
\text { attending technique } \\
\text { permanently }\end{array}$} & $\begin{array}{c}\text { Supreme } \\
\text { management }=83\end{array}$ & 64 & 11 & 8 & 89.16 & 2.67 & agreeing & \multirow{3}{*}{$15.72 *$} \\
\hline & & $\begin{array}{c}\text { Middle } \\
\text { management }=16\end{array}$ & 9 & 1 & 6 & 72.92 & 2.19 & $\begin{array}{c}\text { to a certain } \\
\text { degree }\end{array}$ & \\
\hline & & $\begin{array}{c}\text { Executive } \\
\text { management }=89\end{array}$ & 58 & 4 & 27 & 78.28 & 2.35 & agreeing & \\
\hline & & All $=188$ & 131 & 16 & 41 & 82.62 & 2.48 & agreeing & 116.76 \\
\hline \multirow{4}{*}{$1 / 4$} & \multirow{4}{*}{$\begin{array}{l}\text { Planning for dealing } \\
\text { with restrictions of } \\
\text { using new techniques }\end{array}$} & $\begin{array}{c}\text { Supreme } \\
\text { management }=83\end{array}$ & 67 & 8 & 8 & 90.36 & 2.71 & agreeing & \multirow{3}{*}{$13.18 *$} \\
\hline & & $\begin{array}{c}\text { Middle } \\
\text { management }=16\end{array}$ & 10 & 1 & 5 & 77.08 & 2.31 & $\begin{array}{l}\text { to a certain } \\
\text { degree }\end{array}$ & \\
\hline & & $\begin{array}{c}\text { Executive } \\
\text { management }=89\end{array}$ & 51 & 13 & 25 & 76.40 & 2.29 & $\begin{array}{l}\text { to a certain } \\
\text { degree }\end{array}$ & \\
\hline & & All $=188$ & 128 & 22 & 38 & 82.62 & 2.48 & agreeing & 104.21 \\
\hline \multirow{4}{*}{2} & \multirow{4}{*}{$\begin{array}{c}\text { The club's } \\
\text { organizational structure } \\
\text { agrees with digital } \\
\text { transformation } \\
\text { application }\end{array}$} & $\begin{array}{c}\text { Supreme } \\
\text { management }=83\end{array}$ & 59 & 12 & 12 & 85.54 & 2.57 & agreeing & \multirow{3}{*}{$14.58 *$} \\
\hline & & $\begin{array}{c}\text { Middle } \\
\text { management }=16\end{array}$ & 6 & 3 & 7 & 64.58 & 1.94 & $\begin{array}{l}\text { to a certain } \\
\text { degree }\end{array}$ & \\
\hline & & $\begin{array}{c}\text { Executive } \\
\text { management }=89\end{array}$ & 44 & 12 & 33 & 70.79 & 2.12 & $\begin{array}{l}\text { to a certain } \\
\text { degree }\end{array}$ & \\
\hline & & All $=188$ & 109 & 27 & 52 & 76.77 & 2.30 & $\begin{array}{l}\text { to a certain } \\
\text { degree }\end{array}$ & 56.37 \\
\hline \multirow{4}{*}{3} & \multirow{4}{*}{$\begin{array}{c}\text { There is a specialized } \\
\text { committee or unit for } \\
\text { applying digital } \\
\text { transformation as } \\
\text { planned }\end{array}$} & $\begin{array}{c}\text { Supreme } \\
\text { management }=83\end{array}$ & 60 & 15 & 8 & 87.55 & 2.63 & Agreeing & \multirow{3}{*}{$15.41 *$} \\
\hline & & $\begin{array}{c}\text { Middle } \\
\text { management }=16\end{array}$ & 7 & 3 & 6 & 68.75 & 2.06 & $\begin{array}{l}\text { to a certain } \\
\text { degree }\end{array}$ & \\
\hline & & $\begin{array}{c}\text { Executive } \\
\text { management }=89\end{array}$ & 48 & 12 & 29 & 73.78 & 2.21 & $\begin{array}{l}\text { to a certain } \\
\text { degree }\end{array}$ & \\
\hline & & All $=\mathbf{1 8 8}$ & 115 & 30 & 43 & 79.43 & 2.38 & agreeing & 66.90 \\
\hline \multirow{4}{*}{4} & \multirow{4}{*}{$\begin{array}{l}\text { The club documents } \\
\text { services and transforms } \\
\text { paper forms to } \\
\text { electronic ones }\end{array}$} & $\begin{array}{c}\text { Supreme } \\
\text { management }=83\end{array}$ & 56 & 4 & 23 & 79.92 & 2.40 & agreeing & \multirow{3}{*}{8.82} \\
\hline & & $\begin{array}{c}\text { Middle } \\
\text { management }=16\end{array}$ & 7 & 2 & 7 & 66.67 & 2.00 & $\begin{array}{l}\text { to a certain } \\
\text { degree }\end{array}$ & \\
\hline & & $\begin{array}{c}\text { Executive } \\
\text { management }=89\end{array}$ & 45 & 14 & 30 & 72.28 & 2.17 & $\begin{array}{l}\text { to a certain } \\
\text { degree }\end{array}$ & \\
\hline & & All $=188$ & 108 & 20 & 60 & 75.18 & 2.26 & $\begin{array}{l}\text { to a certain } \\
\text { degree }\end{array}$ & 61.96 \\
\hline \multirow{2}{*}{5} & \multirow{2}{*}{$\begin{array}{l}\text { Information systems } \\
\text { used by the club are } \\
\text { integrated and } \\
\text { associated with all the }\end{array}$} & $\begin{array}{c}\text { Supreme } \\
\text { management }=83\end{array}$ & 51 & 8 & 24 & 77.51 & 2.33 & $\begin{array}{l}\text { to a certain } \\
\text { degree }\end{array}$ & \multirow{2}{*}{5.72} \\
\hline & & $\begin{array}{c}\text { Middle } \\
\text { management }=16\end{array}$ & 6 & 2 & 8 & 62.50 & 1.88 & $\begin{array}{l}\text { to a certain } \\
\text { degree }\end{array}$ & \\
\hline
\end{tabular}




\begin{tabular}{|c|c|c|c|c|c|c|c|c|c|}
\hline $\mathbf{S}$ & Statement content & Departments & Agreeing & $\begin{array}{c}\text { To } \\
\text { a certain } \\
\text { degree }\end{array}$ & Disagreeing & $\begin{array}{c}\text { Out } \\
\text { weighted } \\
\text { percentage }\end{array}$ & Average & Attitude & Chi square \\
\hline & club's units & $\begin{array}{c}\text { Executive } \\
\text { management= } 89\end{array}$ & 41 & 12 & 36 & 68.54 & 2.06 & $\begin{array}{c}\text { to a certain } \\
\text { degree }\end{array}$ & \\
\hline & & All $=188$ & 98 & 22 & 68 & 71.99 & 2.16 & $\begin{array}{l}\text { to a certain } \\
\text { degree }\end{array}$ & 46.77 \\
\hline \multirow{4}{*}{6} & \multirow{4}{*}{$\begin{array}{l}\text { Consulting investment } \\
\text { authorities and experts } \\
\text { to give information } \\
\text { about developing } \\
\text { information technology } \\
\text { fields }\end{array}$} & $\begin{array}{c}\text { Supreme } \\
\text { management }=83\end{array}$ & 60 & 2 & 21 & 82.33 & 2.47 & agreeing & \multirow{3}{*}{$15.35 *$} \\
\hline & & $\begin{array}{c}\text { Middle } \\
\text { management }=16\end{array}$ & 5 & 3 & 8 & 60.42 & 1.81 & $\begin{array}{c}\text { to a certain } \\
\text { degree }\end{array}$ & \\
\hline & & $\begin{array}{c}\text { Executive } \\
\text { management= } 89\end{array}$ & 46 & 9 & 34 & 71.16 & 2.13 & $\begin{array}{c}\text { to a certain } \\
\text { degree }\end{array}$ & \\
\hline & & All $=188$ & 111 & 14 & 63 & 75.18 & 2.26 & $\begin{array}{l}\text { to a certain } \\
\text { degree }\end{array}$ & 75.07 \\
\hline \multirow{4}{*}{7} & \multirow{4}{*}{$\begin{array}{l}\text { The club has a system } \\
\text { for evaluating } \\
\text { employees performance } \\
\text { indicating clear norms } \\
\text { of their ability to use } \\
\text { information technology } \\
\text { applications }\end{array}$} & $\begin{array}{c}\text { Supreme } \\
\text { management }=83\end{array}$ & 50 & 9 & 24 & 77.11 & 2.31 & $\begin{array}{c}\text { to a certain } \\
\text { degree }\end{array}$ & \multirow{3}{*}{5.43} \\
\hline & & $\begin{array}{c}\text { Middle } \\
\text { management }=16\end{array}$ & 5 & 2 & 9 & 58.33 & 1.75 & $\begin{array}{l}\text { to a certain } \\
\text { degree }\end{array}$ & \\
\hline & & $\begin{array}{c}\text { Executive } \\
\text { management }=89\end{array}$ & 49 & 12 & 28 & 74.53 & 2.24 & $\begin{array}{c}\text { to a certain } \\
\text { degree }\end{array}$ & \\
\hline & & All $=188$ & 104 & 23 & 61 & 74.29 & 2.23 & $\begin{array}{l}\text { to a certain } \\
\text { degree }\end{array}$ & 52.41 \\
\hline
\end{tabular}

Chi square value $(0,05,4)=9,488$, Chi square value $(0,05,2)=5,991$

Outweighed arithmetic mean: disagreeing (1: 1.66), to a certain degree (1.67: 2.33), agreeing (2.34:3)

From table (4) agreement of research sample groups on statements number $(1 / 1,4,5,7)$ was clear as the outweighed percentage of the whole sample ranged between (71.99: 84.93) as chi square ranged between (5.43: 8.82)

Results of study of Doaa Elhasban, Weaam Elhayek (2017) (13) indicate the importance of appointing an authority responsible for supervising and following up digital transformation.

Saad Shalaby and Abdellatif Bokhary (2008) (35) confirm that clubs delaying in using new technologies will lag behind other clubs.

Also the above table results proved difference between opinions of the research sample groups on statements number $(\mathbf{1 / 2}, \mathbf{1 / 3}, \mathbf{1 / 4}, \mathbf{2}, \mathbf{3}, \mathbf{6})$ at level $(0.05)$ as chi square ranged between $\left(13.18^{*}: 15.72 *\right)$ with outweighed percentage (75.18: 82.62).

Results of study of Mahmoud Ibrahim and Bassma Haddad (2018) (25), and Ashour Abdelkarim (2010) (9) found that digital transformation depends on compiling a clear strategy by some field expert.

The results of the study of Ashour Abdul Karim (2010) (9) indicate that the development of the level of employees mainly requires a review of their competence, by integrating the human resources of the institutions within the programs of raising the qualification level, with the aim of compatibility with the developments and the reality of these institutions in light of the digital transformation strategy.

The researcher concludes that the sports club management must develop plans and strategies that support the digital transformation system, the more accurately and clearly formulated, the greater the success rate of its implementation. 
Table (5) First Factor Results: Digital Transformation Requirements to Achieve a Competitive Advantage at Clubs (Second Dimension: Digital Skills) (A- Main Skills)

\begin{tabular}{|c|c|c|c|c|c|c|c|c|c|}
\hline $\mathbf{S}$ & Statement content & Departments & Agreeing & $\begin{array}{c}\text { To a } \\
\text { certain } \\
\text { degree }\end{array}$ & Disagreeing & $\begin{array}{l}\text { Out weighted } \\
\text { percentage }\end{array}$ & Average & Attitude & $\begin{array}{l}\text { Chi } \\
\text { square }\end{array}$ \\
\hline 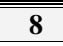 & the club's employees & ave the main digital & 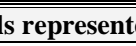 & & & & & & \multirow{4}{*}{3.63} \\
\hline \multirow{4}{*}{$8 / 1$} & \multirow{4}{*}{ Using keyboard } & $\begin{array}{c}\text { Supreme } \\
\text { management }=83\end{array}$ & 68 & 7 & 8 & 90.76 & 2.72 & agreeing & \\
\hline & & $\begin{array}{c}\text { Middle } \\
\text { management }=16\end{array}$ & 10 & 2 & 4 & 79.17 & 2.38 & agreeing & \\
\hline & & $\begin{array}{c}\text { Executive } \\
\text { management }=89\end{array}$ & 71 & 8 & 10 & 89.51 & 2.69 & agreeing & \\
\hline & & All $=188$ & 149 & 17 & 22 & 89.18 & 2.68 & agreeing & 178.61 \\
\hline \multirow{4}{*}{$8 / 2$} & \multirow{4}{*}{$\begin{array}{l}\text { Administrating } \\
\text { files in computers }\end{array}$} & $\begin{array}{c}\text { Supreme } \\
\text { management }=83\end{array}$ & 66 & 8 & 9 & 89.56 & 2.69 & agreeing & \multirow{3}{*}{8.34} \\
\hline & & $\begin{array}{c}\text { Middle } \\
\text { management }=16\end{array}$ & 9 & 1 & 6 & 72.92 & 2.19 & $\begin{array}{c}\text { to a certain } \\
\text { degree }\end{array}$ & \\
\hline & & $\begin{array}{c}\text { Executive } \\
\text { management }=89\end{array}$ & 69 & 9 & 11 & 88.39 & 2.65 & agreeing & \\
\hline & & All $=188$ & 144 & 18 & 26 & 87.59 & 2.63 & agreeing & 158.85 \\
\hline \multirow{4}{*}{$8 / 3$} & \multirow{4}{*}{$\begin{array}{l}\text { Protecting personal } \\
\text { and private data }\end{array}$} & $\begin{array}{c}\text { Supreme } \\
\text { management }=83\end{array}$ & 61 & 11 & 11 & 86.75 & 2.60 & agreeing & \multirow{3}{*}{4.02} \\
\hline & & $\begin{array}{c}\text { Middle } \\
\text { management }=16\end{array}$ & 8 & 3 & 5 & 72.92 & 2.19 & $\begin{array}{c}\text { to a certain } \\
\text { degree }\end{array}$ & \\
\hline & & $\begin{array}{c}\text { Executive } \\
\text { management }=89\end{array}$ & 62 & 12 & 15 & 84.26 & 2.53 & agreeing & \\
\hline & & All $=188$ & 131 & 26 & 31 & 84.40 & 2.53 & agreeing & 111.97 \\
\hline \multirow{4}{*}{$8 / 4$} & \multirow{4}{*}{ Using email } & $\begin{array}{c}\text { Supreme } \\
\text { management }=83\end{array}$ & 65 & 8 & 10 & 88.76 & 2.66 & agreeing & \multirow{3}{*}{$12.31 *$} \\
\hline & & $\begin{array}{c}\text { Middle } \\
\text { management }=16\end{array}$ & 8 & 1 & 7 & 68.75 & 2.06 & $\begin{array}{c}\text { to a certain } \\
\text { degree }\end{array}$ & \\
\hline & & $\begin{array}{c}\text { Executive } \\
\text { management }=89\end{array}$ & 70 & 9 & 10 & 89.14 & 2.67 & agreeing & \\
\hline & & All $=188$ & 143 & 18 & 27 & 87.23 & 2.62 & agreeing & 155.12 \\
\hline \multirow{4}{*}{$8 / 5$} & \multirow{4}{*}{$\begin{array}{l}\text { Browsing, } \\
\text { research and } \\
\text { assorting data, } \\
\text { information and } \\
\text { legal content }\end{array}$} & $\begin{array}{c}\text { Supreme } \\
\text { management }=83\end{array}$ & 67 & 6 & 10 & 89.56 & 2.69 & agreeing & \multirow{3}{*}{$9.54 *$} \\
\hline & & $\begin{array}{c}\text { Middle } \\
\text { management }=16\end{array}$ & 9 & 1 & 6 & 72.92 & 2.19 & $\begin{array}{c}\text { to a certain } \\
\text { degree }\end{array}$ & \\
\hline & & $\begin{array}{c}\text { Executive } \\
\text { management }=89\end{array}$ & 68 & 11 & 10 & 88.39 & 2.65 & agreeing & \\
\hline & & All $=188$ & 144 & 18 & 26 & 87.59 & 2.63 & agreeing & 101,01 \\
\hline \multirow{4}{*}{$8 / 6$} & \multirow{4}{*}{$\begin{array}{l}\text { Communicating } \\
\text { and working in a } \\
\text { team through } \\
\text { digital techniques }\end{array}$} & $\begin{array}{c}\text { Supreme } \\
\text { management }=\mathbf{8 3}\end{array}$ & 65 & 10 & 8 & 89.56 & 2.69 & agreeing & \multirow[t]{3}{*}{$10.59 *$} \\
\hline & & $\begin{array}{c}\text { Middle } \\
\text { management }=16\end{array}$ & 8 & 2 & 6 & 70.83 & 2.13 & $\begin{array}{r}\text { to a certain } \\
\text { degree }\end{array}$ & \\
\hline & & $\begin{array}{c}\text { Executive } \\
\text { management }=89\end{array}$ & 71 & 6 & 12 & 88.76 & 2.66 & agreeing & \\
\hline & & All $=188$ & 144 & 18 & 26 & 87.59 & 2.63 & agreeing & 101,01 \\
\hline \multirow{4}{*}{$8 / 7$} & \multirow{4}{*}{$\begin{array}{l}\text { Filling an internet } \\
\text { form }\end{array}$} & $\begin{array}{c}\text { Supreme } \\
\text { management }=83\end{array}$ & 66 & 8 & 9 & 89.56 & 2.69 & Agreeing & \multirow[t]{3}{*}{$४, \wedge 9$} \\
\hline & & $\begin{array}{c}\text { Middle } \\
\text { management }=16\end{array}$ & 8 & 3 & 5 & 72.92 & 2.19 & $\begin{array}{r}\text { to a certain } \\
\text { degree }\end{array}$ & \\
\hline & & $\begin{array}{c}\text { Executive } \\
\text { management }=89\end{array}$ & 67 & 11 & 11 & 87.64 & 2.63 & agreeing & \\
\hline & & All $=188$ & 141 & 22 & 25 & 87.23 & 2.62 & agreeing & $1 \leqslant 7,90$ \\
\hline
\end{tabular}


From table (5) there are significant differences between the research groups response on statements number $(8 / 4,8 / 5,8 / 6)$ as chi ranged between $(9.54 *: 12.31 *)$ with outweighed percentage (87.23: 87.59).

Ahmed Ghoneim (2004) states the main skills to be enjoyed by employees to achieve digital transformation including information techniques and computers (7: 73).

The sample opinions agreed upon statements number $(8 / 1,8 / 2,8 / 3,8 / 7)$

Mohamed Fathy (2008) refers to efficiencies, knowledge and skills needed by employees enabling them to use advanced knowledge and technology in fulfilling their tasks (27: 14)

The researcher believes that basic digital skills related to the use of digital devices and Internet applications have become an essential component of a new set of skills that must be available to club employees in the digital era.

\section{Table (6) Results of First Factor: Requirements of Digital Transfer to Achieve a Competitive Advantages at Clubs) (second Dimension: Digital Skills)}

\section{(B- Average Skills)}

\begin{tabular}{|c|c|c|c|c|c|c|c|c|c|}
\hline $\begin{array}{l}\text { Chi } \\
\text { square }\end{array}$ & Attitude & Average & $\begin{array}{c}\text { Out } \\
\text { weighted } \\
\text { percentage }\end{array}$ & Disagreeing & $\begin{array}{c}\text { To a } \\
\text { certain } \\
\text { degree }\end{array}$ & Agreeing & Departments & $\begin{array}{c}\text { Statement } \\
\text { content }\end{array}$ & $\mathbf{S}$ \\
\hline \multicolumn{9}{|c|}{ the club's employees has the following average digital skills } & 8 \\
\hline \multirow{3}{*}{5.45} & Agreeing & 2.47 & 82.33 & 18 & 8 & 57 & $\begin{array}{c}\text { Supreme } \\
\text { management }=83\end{array}$ & \multirow{4}{*}{$\begin{array}{l}\text { Evaluating and } \\
\text { managing data, } \\
\text { information and } \\
\text { digital contents }\end{array}$} & \multirow{4}{*}{$8 / 8$} \\
\hline & $\begin{array}{c}\text { to a certain } \\
\text { degree }\end{array}$ & 2.06 & 68.75 & 6 & 3 & 7 & $\begin{array}{c}\text { Middle } \\
\text { management }=16\end{array}$ & & \\
\hline & agreeing & 2.55 & 85.02 & 16 & 8 & 65 & $\begin{array}{c}\text { Executive } \\
\text { management }=89\end{array}$ & & \\
\hline 108.84 & agreeing & 2.47 & 82.45 & 40 & 19 & 129 & All $=188$ & & \\
\hline \multirow{3}{*}{7.38} & $\begin{array}{c}\text { to a certain } \\
\text { degree }\end{array}$ & 2.27 & 75.50 & 23 & 15 & 45 & $\begin{array}{c}\text { Supreme } \\
\text { management }=83\end{array}$ & \multirow{4}{*}{$\begin{array}{l}\text { Digital data } \\
\text { design }\end{array}$} & \multirow{4}{*}{$8 / 9$} \\
\hline & $\begin{array}{c}\text { to a certain } \\
\text { degree }\end{array}$ & 1.88 & 62.50 & 8 & 2 & 6 & $\begin{array}{c}\text { Middle } \\
\text { management }=16\end{array}$ & & \\
\hline & Agreeing & 2.45 & 81.65 & 18 & 13 & 58 & $\begin{array}{c}\text { Executive } \\
\text { management }=89\end{array}$ & & \\
\hline 54.27 & $\begin{array}{l}\text { to a certain } \\
\text { degree }\end{array}$ & 2.32 & 77.30 & 49 & 30 & 109 & All $=188$ & & \\
\hline \multirow{3}{*}{5.43} & $\begin{array}{c}\text { to a certain } \\
\text { degree }\end{array}$ & 2.34 & 77.91 & 21 & 13 & 49 & $\begin{array}{c}\text { Supreme } \\
\text { management }=83\end{array}$ & \multirow{4}{*}{ Data analysis } & \multirow{4}{*}{$8 / 10$} \\
\hline & $\begin{array}{c}\text { to a certain } \\
\text { degree }\end{array}$ & 1.88 & 62.50 & 8 & 2 & 6 & $\begin{array}{c}\text { Middle } \\
\text { management }=16\end{array}$ & & \\
\hline & Agreeing & 2.38 & 79.40 & 20 & 15 & 54 & $\begin{array}{c}\text { Executive } \\
\text { management }=89\end{array}$ & & \\
\hline 54.27 & $\begin{array}{l}\text { to a certain } \\
\text { degree }\end{array}$ & 2.32 & 77.30 & 49 & 30 & 109 & All $=188$ & & \\
\hline \multirow{3}{*}{6.81} & Agreeing & 2.36 & 78.41 & 22 & 9 & 52 & $\begin{array}{c}\text { Supreme } \\
\text { management }=83\end{array}$ & \multirow{4}{*}{$\begin{array}{c}\text { Digital } \\
\text { marketing }\end{array}$} & \multirow{4}{*}{$8 / 11$} \\
\hline & $\begin{array}{c}\text { to a certain } \\
\text { degree }\end{array}$ & 1.88 & 62.50 & 7 & 4 & 5 & $\begin{array}{c}\text { Middle } \\
\text { management }=16\end{array}$ & & \\
\hline & Agreeing & 2.38 & 79.40 & 23 & 9 & 57 & $\begin{array}{c}\text { Executive } \\
\text { management }=89\end{array}$ & & \\
\hline 70.26 & $\begin{array}{c}\text { to a certain } \\
\text { degree }\end{array}$ & 2.33 & 77.66 & 52 & 22 & 114 & All $=188$ & & \\
\hline \multirow{3}{*}{8.67} & Agreeing & 2.36 & 78.41 & 20 & 13 & 50 & $\begin{array}{c}\text { Supreme } \\
\text { management }=83\end{array}$ & \multirow{3}{*}{$\begin{array}{c}\text { Solving technical } \\
\text { problems }\end{array}$} & \multirow{3}{*}{$8 / 12$} \\
\hline & $\begin{array}{c}\text { to a certain } \\
\text { degree }\end{array}$ & 1.69 & 56.25 & 9 & 3 & 4 & $\begin{array}{c}\text { Middle } \\
\text { management }=16\end{array}$ & & \\
\hline & $\begin{array}{c}\text { to a certain } \\
\text { degree }\end{array}$ & 2.28 & 76.03 & 27 & 10 & 52 & $\begin{array}{c}\text { Executive } \\
\text { management }=89\end{array}$ & & \\
\hline
\end{tabular}




\begin{tabular}{|c|c|c|c|c|c|c|c|c|c|}
\hline $\begin{array}{c}\text { Chi } \\
\text { square }\end{array}$ & Attitude & Average & $\begin{array}{c}\text { Out } \\
\text { weighted } \\
\text { percentage }\end{array}$ & Disagreeing & $\begin{array}{c}\text { To a } \\
\text { certain } \\
\text { degree }\end{array}$ & Agreeing & Departments & $\begin{array}{c}\text { Statement } \\
\text { content }\end{array}$ & $\mathbf{S}$ \\
\hline 52.13 & $\begin{array}{c}\text { to a certain } \\
\text { degree }\end{array}$ & 2.27 & 75.53 & 56 & 26 & 106 & All $=188$ & & \\
\hline
\end{tabular}

From table (6) the research subjects responses agreed upon all statements as chi square ranged between (5.43: 8.67) with an outweighed percentage (77.66: 82.45)

International communication association (2018) mentions that average skills enabling us to use digital techniques are more profitable and feasible (23: 7).

The researcher believes that with the increase of knowledge and skills that the beneficiary acquires from the services in the clubs, the club's inability to match the benefactor increases, which requires more qualification and training for club employees. Table (7) First Factor Results: Digital Transformation Requirements to Achieve the Best
Competitive Advantage at Clubs (Second Dimension: Digital Skills)

(C-Advanced Skills)

\begin{tabular}{|c|c|c|c|c|c|c|c|c|c|}
\hline $\begin{array}{l}\text { Chi } \\
\text { square }\end{array}$ & Attitude & Average & $\begin{array}{c}\text { Out } \\
\text { weighted } \\
\text { percentage }\end{array}$ & Disagreeing & $\begin{array}{c}\text { To a } \\
\text { certain } \\
\text { degree }\end{array}$ & Agreeing & Departments & Statement content & $\mathbf{S}$ \\
\hline \multicolumn{9}{|c|}{ the club's employees have the following advanced digital skills : } & 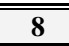 \\
\hline \multirow{3}{*}{$12.48 *$} & $\begin{array}{c}\text { to a certain } \\
\text { degree }\end{array}$ & 2.19 & 73.09 & 25 & 17 & 41 & $\begin{array}{c}\text { Supreme } \\
\text { management }=83\end{array}$ & \multirow{4}{*}{$\begin{array}{l}\text { Computerized } \\
\text { programming }\end{array}$} & \multirow{4}{*}{$8 / 13$} \\
\hline & disagreeing & 1.63 & 54.17 & 9 & 4 & 3 & $\begin{array}{c}\text { Middle } \\
\text { management }=16\end{array}$ & & \\
\hline & $\begin{array}{c}\text { to a certain } \\
\text { degree }\end{array}$ & 1.88 & 62.55 & 32 & 6 & 41 & $\begin{array}{c}\text { Executive } \\
\text { management }=89\end{array}$ & & \\
\hline 28.44 & $\begin{array}{c}\text { to a certain } \\
\text { degree }\end{array}$ & 1.99 & 66.49 & 66 & 27 & 85 & All $=188$ & & \\
\hline \multirow{3}{*}{6.29} & $\begin{array}{c}\text { to a certain } \\
\text { degree }\end{array}$ & 2.07 & 69.08 & 29 & 19 & 35 & $\begin{array}{c}\text { Supreme } \\
\text { management }=83\end{array}$ & \multirow{4}{*}{$\begin{array}{c}\text { Networks } \\
\text { management }\end{array}$} & \multirow{4}{*}{$8 / 14$} \\
\hline & disagreeing & 1.56 & 52.08 & 10 & 3 & 3 & $\begin{array}{c}\text { Middle } \\
\text { management }=16\end{array}$ & & \\
\hline & $\begin{array}{c}\text { to a certain } \\
\text { degree }\end{array}$ & 2.02 & 67.42 & 37 & 13 & 39 & $\begin{array}{c}\text { Executive } \\
\text { management }=89\end{array}$ & & \\
\hline 18.33 & $\begin{array}{l}\text { to a certain } \\
\text { degree }\end{array}$ & 2.01 & 66.84 & 76 & 35 & 77 & All $=188$ & & \\
\hline \multirow{3}{*}{6.64} & $\begin{array}{c}\text { to a certain } \\
\text { degree }\end{array}$ & 2.19 & 73.09 & 26 & 15 & 42 & $\begin{array}{c}\text { Supreme } \\
\text { management }=83\end{array}$ & \multirow{4}{*}{$\begin{array}{c}\text { Developing digital } \\
\text { content }\end{array}$} & \multirow{4}{*}{$8 / 15$} \\
\hline & disagreeing & 1.63 & 54.17 & 10 & 2 & 4 & $\begin{array}{c}\text { Middle } \\
\text { management }=16\end{array}$ & & \\
\hline & $\begin{array}{c}\text { to a certain } \\
\text { degree }\end{array}$ & 1.97 & 65.54 & 39 & 14 & 36 & $\begin{array}{c}\text { Executive } \\
\text { management }=89\end{array}$ & & \\
\hline 24.39 & $\begin{array}{l}\text { to a certain } \\
\text { degree }\end{array}$ & 2.04 & 67.91 & 75 & 31 & 82 & All $=188$ & & \\
\hline \multirow{3}{*}{2.35} & $\begin{array}{c}\begin{array}{c}\text { to a certain } \\
\text { degree }\end{array} \\
\end{array}$ & 2.06 & 68.67 & 31 & 16 & 36 & $\begin{array}{c}\text { Supreme } \\
\text { management }=83 \\
\end{array}$ & \multirow{4}{*}{$\begin{array}{l}\text { Ability to create in } \\
\text { using digital } \\
\text { techniques }\end{array}$} & \multirow{4}{*}{$8 / 16$} \\
\hline & $\begin{array}{c}\text { to a certain } \\
\text { degree }\end{array}$ & 1.69 & 56.25 & 9 & 3 & 4 & $\begin{array}{c}\text { Middle } \\
\text { management }=16\end{array}$ & & \\
\hline & $\begin{array}{c}\text { to a certain } \\
\text { degree }\end{array}$ & 2.01 & 67.04 & 36 & 16 & 37 & $\begin{array}{c}\text { Executive } \\
\text { management= 89 }\end{array}$ & & \\
\hline 18.33 & $\begin{array}{l}\text { to a certain } \\
\text { degree }\end{array}$ & 2.01 & 66.84 & 76 & 35 & 77 & All $=188$ & & \\
\hline
\end{tabular}

From table from table (7) it is clear that the research sample groups response to all statements 
agreed with an outweighed percentage between $(66.48: 67.91)$ and chi square between $(2.35$ : 6.64) with a dominant attitude disagreeing and to a certain degree upon that employees have no digital skills like managing networks, developing digital content.

But for statement number (8/13) related to enjoying digital skills by employees such as computerized programming, opinions of supreme and executive administration were to a certain degree

Communication international association (2018) mentions that advanced skills are skills needed by specialists in information technology and communication such as computerized programming and networks management (23:9).

The researcher believes that digital skills are constantly evolving with changes in technology, allowing policymakers and digital skills providers to ensure the continued relevance and up-todatedness of their programs and training curricula.

Table (8) First Factor Results: Digital Transformation Requirements to Achieve the Best Competitive Advantage at Clubs (Third Dimension: Digital Techniques) (A- Things Internet)

\begin{tabular}{|c|c|c|c|c|c|c|c|c|c|}
\hline $\begin{array}{l}\text { Chi } \\
\text { square }\end{array}$ & Attitude & Average & $\begin{array}{c}\text { Out } \\
\text { weighted } \\
\text { percentage }\end{array}$ & Disagreeing & $\begin{array}{c}\text { To a } \\
\text { certain } \\
\text { degree }\end{array}$ & Agreeing & Departments & Statement content & $\mathbf{S}$ \\
\hline \multirow{3}{*}{8.36} & agreeing & 2.65 & 88.35 & 11 & 7 & 65 & $\begin{array}{c}\text { Supreme } \\
\text { management }=83\end{array}$ & \multirow{4}{*}{$\begin{array}{c}\text { The club has } \\
\text { updated and high } \\
\text { speed internet lines }\end{array}$} & \multirow{4}{*}{9} \\
\hline & $\begin{array}{c}\text { to a } \\
\text { certain } \\
\text { degree }\end{array}$ & 2.13 & 70.83 & 6 & 2 & 8 & $\begin{array}{c}\text { Middle } \\
\text { management }=16\end{array}$ & & \\
\hline & agreeing & 2.46 & 82.02 & 17 & 14 & 58 & $\begin{array}{c}\text { Executive } \\
\text { management }=89\end{array}$ & & \\
\hline 112.73 & agreeing & 2.52 & 83.87 & 34 & 23 & 131 & All $=188$ & & \\
\hline \multirow{3}{*}{$15.36 *$} & agreeing & 2.75 & 91.57 & 8 & 5 & 70 & $\begin{array}{c}\text { Supreme } \\
\text { management }=83\end{array}$ & \multirow{4}{*}{$\begin{array}{l}\text { There is a link } \\
\text { between internet } \\
\text { and intranet }\end{array}$} & \multirow{4}{*}{10} \\
\hline & $\begin{array}{c}\text { to a } \\
\text { certain } \\
\text { degree }\end{array}$ & 2.19 & 72.92 & 6 & 1 & 9 & $\begin{array}{c}\text { Middle } \\
\text { management }=16\end{array}$ & & \\
\hline & agreeing & 2.43 & 80.90 & 18 & 15 & 56 & $\begin{array}{c}\text { Executive } \\
\text { management }=89\end{array}$ & & \\
\hline 126.20 & agreeing & 2.55 & 84.93 & 32 & 21 & 135 & All $=188$ & & \\
\hline \multirow{3}{*}{$9.62 *$} & agreeing & 2.70 & 89.96 & 9 & 7 & 67 & $\begin{array}{c}\text { Supreme } \\
\text { management }=83\end{array}$ & \multirow{4}{*}{$\begin{array}{c}\text { There are strong } \\
\text { anti-hacking } \\
\text { programs }\end{array}$} & \multirow{4}{*}{11} \\
\hline & $\begin{array}{c}\text { to a } \\
\text { certain } \\
\text { degree }\end{array}$ & 2.25 & 75.00 & 5 & 2 & 9 & $\begin{array}{c}\text { Middle } \\
\text { management }=16\end{array}$ & & \\
\hline & agreeing & 2.40 & 80.15 & 19 & 15 & 55 & $\begin{array}{c}\text { Executive } \\
\text { management }=89\end{array}$ & & \\
\hline 112.41 & agreeing & 2.52 & 84.04 & 33 & 24 & 131 & All $=188$ & & \\
\hline \multirow{3}{*}{$13.70 *$} & agreeing & 2.73 & 91.16 & 8 & 6 & 69 & $\begin{array}{c}\text { Supreme } \\
\text { management }=83\end{array}$ & \multirow{4}{*}{$\begin{array}{l}\text { The network can be } \\
\text { technically flexible }\end{array}$} & \multirow{4}{*}{12} \\
\hline & $\begin{array}{c}\text { to a } \\
\text { certain } \\
\text { degree }\end{array}$ & 2.31 & 77.08 & 5 & 1 & 10 & $\begin{array}{c}\text { Middle } \\
\text { management }=16\end{array}$ & & \\
\hline & agreeing & 2.36 & 78.65 & 21 & 15 & 53 & $\begin{array}{c}\text { Executive } \\
\text { management }=89\end{array}$ & & \\
\hline 116.21 & agreeing & 2.52 & 84.04 & 34 & 22 & 132 & All $=188$ & & \\
\hline \multicolumn{9}{|c|}{ the club has a detailed data base about : } & 13 \\
\hline
\end{tabular}




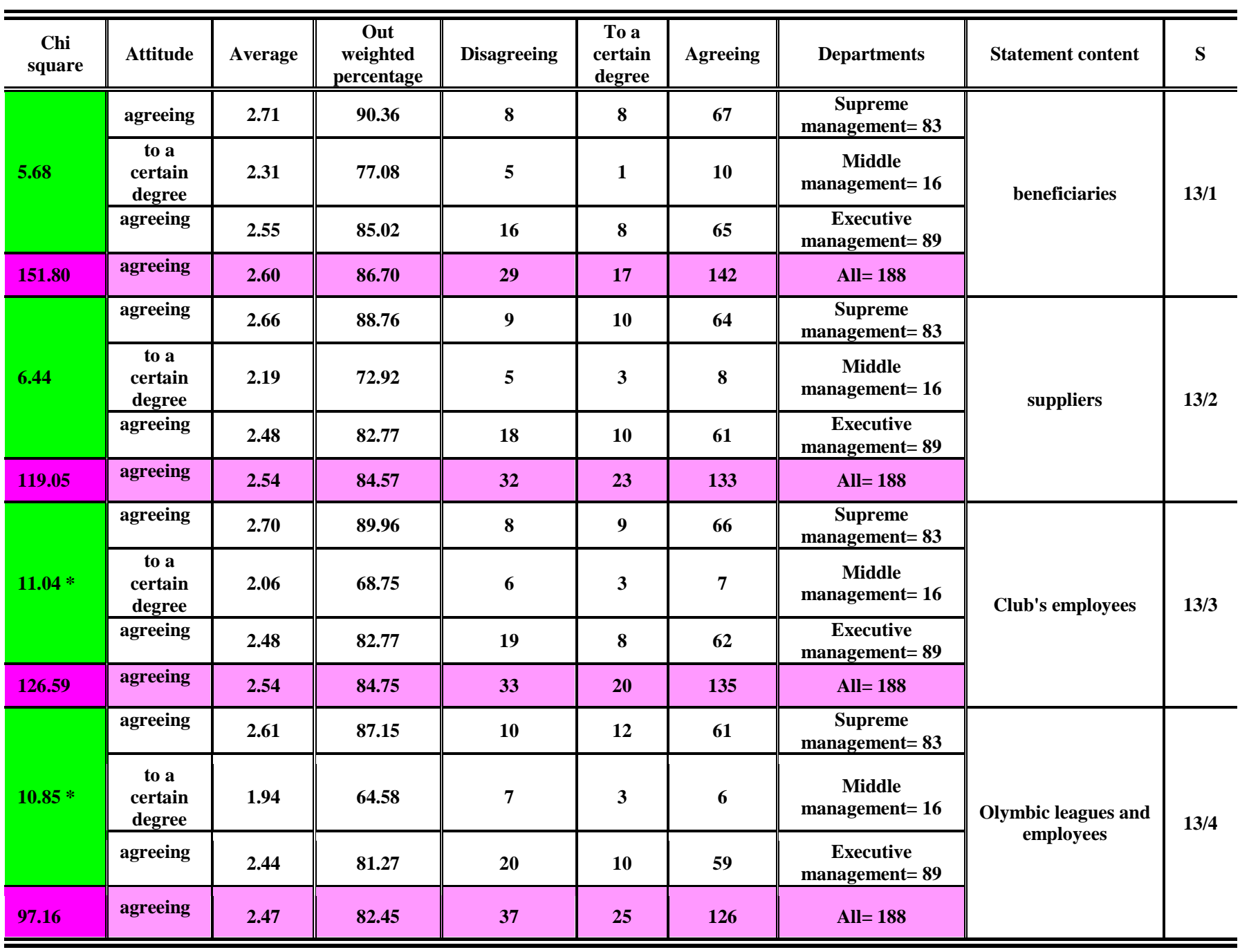

From table (8), it is clear that opinions of the research sample groups agreed upon statements number $(9,13 / 1,13 / 2)$ as the outweighed percentage reached $(83.87: 86.70)$ with chi square between $(5.68: 8.36)$

Results of Hytham Fayez (2016) (17), Ahlam Alfiki (2014) (4) agreed upon the availability of needed infra-structure helping clubs to activate modern electronic services.

But for statements number $(10,11,12,13 / 3,13 / 4)$ the research sample groups' opinion s varied between agreeing and to a certain degree.

Results of study of Tawil Osama (2017) (40) proves lack of security systems of protecting data of sports departments deals.

The researcher concludes that the activation of the Internet of things will contribute to the expansion of better services in sports clubs, which requires the availability of regular, high-speed Internet services that connect the departments to each other them within the club, as well as allow communication with other parties concerned and not concerned with sports. 
Table (9) First Factor Results: Digital Transformation Requirements to Achieve the Best Competitive Advantage at Clubs (Third Dimension: Digital Techniques) (B-Cloud computing)

\begin{tabular}{|c|c|c|c|c|c|c|c|c|c|}
\hline $\begin{array}{l}\text { Chi } \\
\text { square }\end{array}$ & Attitude & Average & $\begin{array}{c}\text { Out } \\
\text { weighted } \\
\text { percentage }\end{array}$ & Disagreeing & $\begin{array}{c}\text { To a } \\
\text { certain } \\
\text { degree }\end{array}$ & Agreeing & Departments & Statement content & $\mathbf{S}$ \\
\hline \multirow{3}{*}{$11.79 *$} & agreeing & 2.66 & 88.76 & 9 & 10 & 64 & $\begin{array}{c}\text { Supreme management }= \\
83\end{array}$ & \multirow{4}{*}{$\begin{array}{l}\text { The club uses cloud } \\
\text { computerization } \\
\text { services and } \\
\text { applications supplied } \\
\text { by service providers } \\
\text { such as Google, } \\
\text { Microsoft and Amazon }\end{array}$} & \multirow{4}{*}{14} \\
\hline & $\begin{array}{c}\text { to a } \\
\text { certain } \\
\text { degree }\end{array}$ & 2.25 & 75.00 & 5 & 2 & 9 & $\underset{16}{\text { Middle management }=}$ & & \\
\hline & $\begin{array}{l}\text { to a } \\
\text { certain } \\
\text { degree }\end{array}$ & 2.28 & 76.03 & 23 & 18 & 48 & $\begin{array}{c}\text { Executive } \\
\text { management }=89\end{array}$ & & \\
\hline 81.84 & agreeing & 2.45 & 81.56 & 37 & 30 & 121 & All $=188$ & & \\
\hline \multirow{3}{*}{$10.45 *$} & agreeing & 2.52 & 83.94 & 12 & 16 & 55 & $\begin{array}{l}\text { Supreme management }= \\
83\end{array}$ & \multirow{4}{*}{$\begin{array}{l}\text { Using cloud } \\
\text { computerization agrees } \\
\text { with the club's } \\
\text { activities }\end{array}$} & \multirow{4}{*}{15} \\
\hline & $\begin{array}{l}\text { to a } \\
\text { certain } \\
\text { degree }\end{array}$ & 1.94 & 64.58 & 7 & 3 & 6 & $\begin{array}{l}\text { Middle management }= \\
16\end{array}$ & & \\
\hline & $\begin{array}{c}\text { to a } \\
\text { certain } \\
\text { degree }\end{array}$ & 2.19 & 73.03 & 28 & 16 & 45 & $\begin{array}{c}\text { Executive } \\
\text { management }=89\end{array}$ & & \\
\hline 46.10 & $\begin{array}{c}\text { to a } \\
\text { certain } \\
\text { degree }\end{array}$ & 2.31 & 77.13 & 47 & 35 & 106 & All $=188$ & & \\
\hline \multirow{3}{*}{$10.31 *$} & agreeing & 2.58 & 85.94 & 11 & 13 & 59 & $\begin{array}{l}\text { Supreme management }= \\
83\end{array}$ & \multirow{4}{*}{$\begin{array}{l}\text { Cloud computerization } \\
\text { are used to reach } \\
\text { applications, resources, } \\
\text { data and information } \\
\text { on time }\end{array}$} & \multirow{4}{*}{16} \\
\hline & $\begin{array}{c}\text { to a } \\
\text { certain } \\
\text { degree }\end{array}$ & 2.00 & 66.67 & 7 & 2 & 7 & $\underset{16}{\text { Middle management }}=$ & & \\
\hline & $\begin{array}{l}\text { to a } \\
\text { certain } \\
\text { degree }\end{array}$ & 2.27 & 75.66 & 25 & 15 & 49 & $\begin{array}{c}\text { Executive } \\
\text { management= } 89\end{array}$ & & \\
\hline 66.90 & agreeing & 2.38 & 79.43 & 43 & 30 & 115 & All $=188$ & & \\
\hline \multirow{3}{*}{$14.44 *$} & agreeing & 2.63 & 87.55 & 9 & 13 & 61 & $\begin{array}{c}\text { Supreme management= } \\
83\end{array}$ & \multirow{4}{*}{$\begin{array}{l}\text { Cloud computerization } \\
\text { develops mechanism } \\
\text { and means of handling } \\
\text { and sharing } \\
\text { information }\end{array}$} & \multirow{4}{*}{17} \\
\hline & $\begin{array}{l}\text { to a } \\
\text { certain } \\
\text { degree }\end{array}$ & 2.06 & 68.75 & 6 & 3 & 7 & $\begin{array}{l}\text { Middle management= } \\
16\end{array}$ & & \\
\hline & $\begin{array}{l}\text { to a } \\
\text { certain } \\
\text { degree }\end{array}$ & 2.19 & 73.3 & 29 & 14 & 46 & $\begin{array}{c}\text { Executive } \\
\text { management }=89\end{array}$ & & \\
\hline 64.64 & agreeing & 2.37 & 79.08 & 44 & 30 & 114 & All $=188$ & & \\
\hline \multirow{3}{*}{$20.47 *$} & agreeing & 2.64 & 87.95 & 10 & 10 & 63 & $\begin{array}{c}\text { Supreme management= } \\
83\end{array}$ & \multirow{4}{*}{$\begin{array}{l}\text { Information safety } \\
\text { factors are available } \\
\text { for data and files } \\
\text { uploaded to the cloud }\end{array}$} & \multirow{4}{*}{18} \\
\hline & $\begin{array}{l}\text { to a } \\
\text { certain } \\
\text { degree }\end{array}$ & 1.94 & 64.58 & 7 & 3 & 6 & $\underset{16}{\text { Middle management }}=$ & & \\
\hline & $\begin{array}{l}\text { to a } \\
\text { certain } \\
\text { degree }\end{array}$ & 2.10 & 70.04 & 32 & 16 & 41 & $\begin{array}{c}\text { Executive } \\
\text { management }=89\end{array}$ & & \\
\hline 56.82 & $\begin{array}{l}\text { to a } \\
\text { certain } \\
\text { degree }\end{array}$ & 2.32 & 77.48 & 49 & 29 & 110 & All $=188$ & & \\
\hline
\end{tabular}

From table (9), there are statistical significant differences between research groups opinions on all statements as chi square ranged between $\left(10.31^{*}: 20.47^{*}\right)$ and outweighed percentage (77.13: 18.56) 
Results of study of Safaa Soliman (2019) (36), Alia Abdelmoniem (2014) (8) regarding service providers verifying quality of used network, applications and freedom fron any security gaps.

Ahmed Mahgoub (2015) (6) recommends studying service providers in details and identifying security protection supplied by service providers.

The researcher concludes that the use of cloud computing technology in clubs contributes to providing workers with access to computer resources and programs without being bound by time and place limits, and ease of communication between different departments, with the possibility of using them in cloud storage.

Table (10) First Factor Results: Digital Transformation Requirements to Achieve the Best Competitive Advantage at Clubs (Third Dimension: Digital Techniques) (C - Blocks Chain "Blue Kitchen")

\begin{tabular}{|c|c|c|c|c|c|c|c|c|c|}
\hline $\begin{array}{l}\text { Chi } \\
\text { square }\end{array}$ & Attitude & Average & $\begin{array}{c}\text { Out } \\
\text { weighted } \\
\text { percentage }\end{array}$ & Disagreeing & $\begin{array}{c}\text { To a } \\
\text { certain } \\
\text { degree } \\
\end{array}$ & Agreeing & Departments & Statement content & $\mathbf{S}$ \\
\hline \multirow{3}{*}{$10.58 *$} & agreeing & 2.69 & 89.56 & 8 & 10 & 65 & $\begin{array}{c}\text { Supreme } \\
\text { management }=83\end{array}$ & \multirow{4}{*}{$\begin{array}{l}\text { There are } \\
\text { employees } \\
\text { specialized in } \\
\text { information security } \\
\text { and protection }\end{array}$} & \multirow{4}{*}{19} \\
\hline & $\begin{array}{c}\text { to a } \\
\text { certain } \\
\text { degree }\end{array}$ & 2.19 & 72.92 & 4 & 5 & 7 & $\begin{array}{c}\text { Middle } \\
\text { management }=16\end{array}$ & & \\
\hline & agreeing & 2.44 & 81.27 & 19 & 12 & 58 & $\begin{array}{c}\text { Executive } \\
\text { management }=89\end{array}$ & & \\
\hline 108.65 & agreeing & 2.53 & 84.22 & 31 & 27 & 130 & All $=188$ & & \\
\hline \multirow{3}{*}{$12.07 *$} & agreeing & 2.64 & 87.95 & 10 & 10 & 63 & $\begin{array}{c}\text { Supreme } \\
\text { management }=83\end{array}$ & \multirow{4}{*}{$\begin{array}{l}\text { Specialists use } \\
\text { blocks chain to } \\
\text { discover and } \\
\text { describe hacking }\end{array}$} & \multirow{4}{*}{2} \\
\hline & $\begin{array}{c}\text { to a } \\
\text { certain } \\
\text { degree }\end{array}$ & 2.00 & 66.67 & 6 & 6 & 6 & $\begin{array}{c}\text { Middle } \\
\text { management }=16\end{array}$ & & \\
\hline & $\begin{array}{c}\text { to a } \\
\text { certain } \\
\text { degree }\end{array}$ & 2.33 & $\mathbf{7 7 . 5 3}$ & 22 & 51 & 51 & $\begin{array}{c}\text { Executive } \\
\text { management }=89\end{array}$ & & \\
\hline 79.19 & agreeing & 2.44 & 81.21 & 38 & 120 & 120 & All $=188$ & & \\
\hline \multirow{3}{*}{$14.45 *$} & agreeing & 2.69 & 89.56 & 8 & 65 & 65 & $\begin{array}{c}\text { Supreme } \\
\text { management }=83\end{array}$ & \multirow{4}{*}{$\begin{array}{l}\text { There are specialists } \\
\text { in observing } \\
\text { hacking at time of } \\
\text { occurrence and } \\
\text { repairing any } \\
\text { resulting defect }\end{array}$} & \multirow{4}{*}{21} \\
\hline & $\begin{array}{c}\text { to a } \\
\text { certain } \\
\text { degree }\end{array}$ & 2.13 & 70.83 & 4 & 6 & 6 & $\begin{array}{c}\text { Middle } \\
\text { management }=16\end{array}$ & & \\
\hline & agreeing & 2.37 & 79.03 & 20 & 53 & 53 & $\begin{array}{c}\text { Executive } \\
\text { management= } 89\end{array}$ & & \\
\hline 90.4 & agreeing & 2.49 & 82.98 & 32 & 124 & 124 & All $=188$ & & \\
\hline
\end{tabular}

From table (10) there are significant differences between the research group responses on all statements as chi square ranged between $\left(10.58^{*}: 14.45^{*}\right)$ with an outweighed percentage (81.21: 84.22)

Fatma Elsebaey (2019), Ehab Khalifa (2018) (22) mention that blue kitchen techniques has 2 main norms, non-centralism and international transparency in managing all deals (15:4)

The researcher considers the need to work on holding introductory courses for workers in sports clubs to learn about blockchain technology and how to deal with this technology and to urge its application and adoption because of its benefits in preserving data integrity and protecting 
information.

Table (11) First Factor Results: Digital Transformation Requirements to Achieve the Best Competitive Advantage at Clubs (Third Dimension: Digital Techniques) (D - Open Governmental Data)

\begin{tabular}{|c|c|c|c|c|c|c|c|c|c|}
\hline $\begin{array}{l}\text { Chi } \\
\text { square }\end{array}$ & Attitude & Average & $\begin{array}{c}\text { Out } \\
\text { weighted } \\
\text { percentage }\end{array}$ & Disagreeing & $\begin{array}{c}\text { To a } \\
\text { certain } \\
\text { degree } \\
\end{array}$ & Agreeing & Departments & Statement content & $\mathbf{S}$ \\
\hline \multirow{3}{*}{7.42} & agreeing & 2.53 & 84.34 & 13 & 13 & 57 & $\begin{array}{c}\text { Supreme } \\
\text { management }=83\end{array}$ & \multirow{4}{*}{$\begin{array}{l}\text { Open governmental data } \\
\text { are used in developing } \\
\text { the club's administrative } \\
\text { performance via } \\
\text { providing the content } \\
\text { with club's service } \\
\text { related data }\end{array}$} & \multirow{4}{*}{22} \\
\hline & $\begin{array}{c}\text { to a certain } \\
\text { degree }\end{array}$ & 2.06 & 68.75 & 7 & 1 & 8 & $\begin{array}{c}\text { Middle } \\
\text { management }=16\end{array}$ & & \\
\hline & agreeing & 2.35 & 78.28 & 21 & 16 & 52 & $\begin{array}{c}\text { Executive } \\
\text { management= 89 }\end{array}$ & & \\
\hline 71.63 & agreeing & 2.40 & 80.14 & 41 & 30 & 117 & All $=188$ & & \\
\hline \multirow{3}{*}{8.22} & agreeing & 2.52 & 83.94 & 12 & 16 & 55 & $\begin{array}{c}\text { Supreme } \\
\text { management }=83\end{array}$ & \multirow{4}{*}{$\begin{array}{l}\text { The club profits from } \\
\text { open governmental data } \\
\text { in statistical analysis for } \\
\text { a certain subject }\end{array}$} & \multirow{4}{*}{23} \\
\hline & $\begin{array}{l}\text { to a certain } \\
\text { degree }\end{array}$ & 2.00 & 66.67 & 7 & 2 & 7 & $\begin{array}{c}\text { Middle } \\
\text { management= } 16\end{array}$ & & \\
\hline & $\begin{array}{c}\text { to a certain } \\
\text { degree }\end{array}$ & 2.28 & 76.03 & 24 & 16 & 49 & $\begin{array}{c}\text { Executive } \\
\text { management= 89 }\end{array}$ & & \\
\hline 56.56 & agreeing & 2.36 & 78.72 & 43 & 34 & 111 & All $=188$ & & \\
\hline \multirow{3}{*}{7.46} & agreeing & 2.63 & 87.55 & 11 & 9 & 63 & $\begin{array}{c}\text { Supreme } \\
\text { management }=83\end{array}$ & \multirow{4}{*}{$\begin{array}{l}\text { Technical and } \\
\text { technological structure of } \\
\text { sites availing open data } \\
\text { are not enough }\end{array}$} & \multirow{4}{*}{24} \\
\hline & $\begin{array}{c}\text { to a certain } \\
\text { degree }\end{array}$ & 2.06 & 86.75 & 6 & 3 & 7 & $\begin{array}{c}\text { Middle } \\
\text { management }=16\end{array}$ & & \\
\hline & agreeing & 2.47 & 82.40 & 17 & 13 & 59 & $\begin{array}{c}\text { Executive } \\
\text { management }=89\end{array}$ & & \\
\hline 105.79 & agreeing & 2.51 & 83.51 & 34 & 25 & 129 & All $=188$ & & \\
\hline \multirow{3}{*}{6.79} & agreeing & 2.54 & 84.74 & 14 & 10 & 59 & $\begin{array}{c}\text { Supreme } \\
\text { management }=83\end{array}$ & \multirow{4}{*}{$\begin{array}{l}\text { Legal restrictions and } \\
\text { confident information } \\
\text { limit profiting from and } \\
\text { reuse }\end{array}$} & \multirow{4}{*}{25} \\
\hline & $\begin{array}{c}\text { to a certain } \\
\text { degree }\end{array}$ & 2.00 & 66.67 & 6 & 4 & 6 & $\begin{array}{c}\text { Middle } \\
\text { management }=16\end{array}$ & & \\
\hline & agreeing & 2.42 & 80.52 & 20 & 12 & 57 & $\begin{array}{c}\text { Executive } \\
\text { management= 89 }\end{array}$ & & \\
\hline \multirow[t]{2}{*}{85.83} & agreeing & 2.44 & 81.21 & 40 & 26 & 122 & All $=188$ & & \\
\hline & & & & & & & & $\begin{array}{l}\text { The club profits from } \\
\text { data provided by }\end{array}$ & 26 \\
\hline \multirow{3}{*}{8.28} & agreeing & 2.65 & 88.35 & 12 & 5 & 66 & $\begin{array}{c}\text { Supreme } \\
\text { management }=83\end{array}$ & \multirow{4}{*}{$\begin{array}{l}\text { Ministries and } \\
\text { authorities site }\end{array}$} & \multirow{4}{*}{$26 / 1$} \\
\hline & $\begin{array}{l}\text { to a certain } \\
\text { degree }\end{array}$ & 2.13 & 70.83 & 6 & 2 & 8 & $\begin{array}{c}\text { Middle } \\
\text { management= } 16\end{array}$ & & \\
\hline & agreeing & 2.51 & 83.52 & 16 & 12 & 61 & $\begin{array}{c}\text { Executive } \\
\text { management= } 89\end{array}$ & & \\
\hline 127.03 & agreeing & 2.54 & 84.557 & 34 & 19 & 135 & All $=188$ & & \\
\hline \multirow{3}{*}{$10.78 *$} & agreeing & 2.61 & 87.15 & 11 & 10 & 62 & $\begin{array}{c}\text { Supreme } \\
\text { management }=83\end{array}$ & \multirow{4}{*}{$\begin{array}{c}\text { Statistical central } \\
\text { authority }\end{array}$} & \multirow{4}{*}{$26 / 2$} \\
\hline & sometimes & 1.94 & 64.58 & 7 & 3 & 6 & $\begin{array}{c}\text { Middle } \\
\text { management }=16\end{array}$ & & \\
\hline & agreeing & 2.49 & 83.15 & 15 & 15 & 59 & $\begin{array}{c}\text { Executive } \\
\text { management= 89 }\end{array}$ & & \\
\hline 99.27 & agreeing & 2.50 & 83.33 & 33 & 28 & 127 & All $=188$ & & \\
\hline \multirow{2}{*}{$13.45 *$} & agreeing & 2.66 & 88.76 & 13 & 2 & 68 & $\begin{array}{c}\text { Supreme } \\
\text { management }=83\end{array}$ & \multirow{2}{*}{ Sports leagues sites } & \multirow{2}{*}{$26 / 3$} \\
\hline & $\begin{array}{c}\text { to a certain } \\
\text { degree }\end{array}$ & 2.13 & 70.83 & 6 & 2 & 8 & $\begin{array}{c}\text { Middle } \\
\text { management }=16\end{array}$ & & \\
\hline
\end{tabular}




\begin{tabular}{|c|c|c|c|c|c|c|c|c|c|}
\hline $\begin{array}{l}\text { Chi } \\
\text { square }\end{array}$ & Attitude & Average & $\begin{array}{c}\text { Out } \\
\text { weighted } \\
\text { percentage }\end{array}$ & Disagreeing & $\begin{array}{c}\text { To a } \\
\text { certain } \\
\text { degree } \\
\end{array}$ & Agreeing & Departments & Statement content & $\mathbf{S}$ \\
\hline & agreeing & 2.54 & 84.64 & 14 & 13 & 62 & $\begin{array}{c}\text { Executive } \\
\text { management }=89\end{array}$ & & \\
\hline 137.88 & agreeing & 2.56 & 85.28 & 33 & 17 & 138 & All $=188$ & & \\
\hline \multirow{3}{*}{6.32} & agreeing & 2.54 & 84.74 & 15 & 8 & 60 & $\begin{array}{c}\text { Supreme } \\
\text { management }=83\end{array}$ & \multirow{4}{*}{ Olympic committees sites } & \multirow{4}{*}{$26 / 4$} \\
\hline & $\begin{array}{c}\text { to a certain } \\
\text { degree }\end{array}$ & 2.06 & 68.75 & 6 & 3 & 7 & $\begin{array}{c}\text { Middle } \\
\text { management }=16\end{array}$ & & \\
\hline & agreeing & 2.51 & 83.52 & 15 & 14 & 60 & $\begin{array}{c}\text { Executive } \\
\text { management= 89 }\end{array}$ & & \\
\hline 100.03 & agreeing & 2.48 & 82.80 & 36 & 25 & 127 & All $=188$ & & \\
\hline
\end{tabular}

From table (11) there are differences between research groups responses to statements numbers $(26 / 2,26 / 3)$ as chi square reached $\left(10.87^{*}\right),\left(13.45^{*}\right)$ and outweighed percentage (83.33), (85.28) respectively.

As the sample opinions agreed upon statements numbers $(22,23,24,25,26 / 1,26 / 4)$ as chi square ranged between $(6.32: 8.28)$ and outweighed percentage (78.72:84.57)

The results of Khulud Bint Salem Bin Saleh (2019) (24) indicate that open governmental data must be integrated, compiled at the highest level of accuracy, and available to the largest number of users and for the broadest range of purposes without distinction of anyone and without registration conditions, and is not subject to any copyright, patents or trademarks, with easy and quick access to it in a timely manner to preserve its value.

Haroun Abdalla (2009) (19) mentions that creating a data base is the basis of governmental entrance to internet to provide people and investors with the latest information.

The researcher believes that it is necessary to develop and upgrade government databases and establish central repositories for information, considering a specific policy for informational security in accordance to international standards in this regard.

Table (12) First Factor Results: Digital Transformation Requirements to Achieve the Best Competitive Advantage at Clubs (Third Dimension: Digital Techniques) (E- Artificial Intelligence)

\begin{tabular}{|c|c|c|c|c|c|c|c|c|c|}
\hline $\begin{array}{l}\text { Chi } \\
\text { square }\end{array}$ & Attitude & Average & $\begin{array}{c}\text { Out } \\
\text { weighted } \\
\text { percentage }\end{array}$ & Disagreeing & $\begin{array}{c}\text { To a } \\
\text { certain } \\
\text { degree }\end{array}$ & Agreeing & Departments & Statement content & $\mathbf{S}$ \\
\hline & & & & & & & & $\begin{array}{l}\text { Artificial intelligence is } \\
\text { used by clubs in }\end{array}$ & 27 \\
\hline \multirow{3}{*}{8.39} & agreeing & 2.65 & 88.35 & 10 & 9 & 64 & $\begin{array}{c}\text { Supreme } \\
\text { management }=83\end{array}$ & \multirow{4}{*}{$\begin{array}{c}\text { Discovering talents } \\
\text { and expecting and } \\
\text { developing talented to } \\
\text { make sports } \\
\text { champions }\end{array}$} & \multirow{4}{*}{$27 / 1$} \\
\hline & $\begin{array}{c}\text { to a } \\
\text { certain } \\
\text { degree }\end{array}$ & 2.06 & 68.75 & 6 & 3 & 7 & $\begin{array}{c}\text { Middle } \\
\text { management }=16\end{array}$ & & \\
\hline & agreeing & 2.47 & 82.40 & 17 & 13 & 59 & $\begin{array}{c}\text { Executive } \\
\text { management }=89\end{array}$ & & \\
\hline 109.03 & agreeing & 2.52 & 83.87 & 33 & 25 & 130 & All $=188$ & & \\
\hline $14.50 *$ & agreeing & 2.65 & 88.35 & 11 & 7 & 65 & $\begin{array}{c}\text { Supreme } \\
\text { management }=83\end{array}$ & $\begin{array}{c}\text { Developing and } \\
\text { improving athletes }\end{array}$ & $27 / 2$ \\
\hline
\end{tabular}




\begin{tabular}{|c|c|c|c|c|c|c|c|c|c|}
\hline $\begin{array}{l}\text { Chi } \\
\text { square }\end{array}$ & Attitude & Average & $\begin{array}{c}\text { Out } \\
\text { weighted } \\
\text { percentage }\end{array}$ & Disagreeing & $\begin{array}{c}\text { To a } \\
\text { certain } \\
\text { degree }\end{array}$ & Agreeing & Departments & Statement content & $\mathbf{S}$ \\
\hline & $\begin{array}{c}\text { to a } \\
\text { certain } \\
\text { degree }\end{array}$ & 2.06 & 68.75 & 7 & 1 & 8 & $\begin{array}{c}\text { Middle } \\
\text { management }=16\end{array}$ & \multirow{3}{*}{$\begin{array}{l}\text { performance level by } \\
\text { analyzing as great data } \\
\text { of players and teams } \\
\text { performance as } \\
\text { possible }\end{array}$} & \\
\hline & agreeing & 2.44 & 81.27 & 16 & 18 & 55 & $\begin{array}{c}\text { Executive } \\
\text { management }=89\end{array}$ & & \\
\hline 102.68 & agreeing & 2.50 & 83.33 & 34 & 26 & 128 & All $=188$ & & \\
\hline \multirow{3}{*}{$9.86 *$} & agreeing & 2.42 & 80.72 & 18 & 12 & 53 & $\begin{array}{c}\text { Supreme } \\
\text { management }=83\end{array}$ & \multirow{4}{*}{$\begin{array}{l}\text { Shooting scenes with } \\
\text { angle } 360^{\circ} \text { for sports } \\
\text { activities details of } \\
\text { participants' faces } \\
\text {,movements and acts }\end{array}$} & \multirow{4}{*}{$27 / 3$} \\
\hline & $\begin{array}{c}\text { to a } \\
\text { certain } \\
\text { degree }\end{array}$ & 1.88 & 62.50 & 8 & 2 & 6 & $\begin{array}{c}\text { Middle } \\
\text { management }=16\end{array}$ & & \\
\hline & $\begin{array}{c}\text { to a } \\
\text { certain } \\
\text { degree }\end{array}$ & 2.19 & 73.03 & 25 & 22 & 42 & $\begin{array}{c}\text { Executive } \\
\text { management }=89\end{array}$ & & \\
\hline 36.79 & $\begin{array}{c}\text { to a } \\
\text { certain } \\
\text { degree }\end{array}$ & 2.27 & 75.53 & 51 & 36 & 101 & All $=188$ & & \\
\hline \multirow{3}{*}{$15.22 *$} & agreeing & 2.41 & 80.32 & 21 & 7 & 55 & $\begin{array}{c}\text { Supreme } \\
\text { management }=83\end{array}$ & \multirow{4}{*}{$\begin{array}{l}\text { Producing press } \\
\text { reports and videos } \\
\text { clarifying facts } \\
\text { occurred during } \\
\text { different activities }\end{array}$} & \multirow{4}{*}{$27 / 4$} \\
\hline & $\begin{array}{c}\text { to a } \\
\text { certain } \\
\text { degree } \\
\end{array}$ & 1.75 & 58.33 & 9 & 2 & 5 & $\begin{array}{c}\text { Middle } \\
\text { management= }=16\end{array}$ & & \\
\hline & $\begin{array}{c}\text { to a } \\
\text { certain } \\
\text { degree }\end{array}$ & 2.29 & 76.40 & 21 & 21 & 47 & $\begin{array}{c}\text { Executive } \\
\text { management= } 89\end{array}$ & & \\
\hline 50.56 & $\begin{array}{c}\text { to a } \\
\text { certain } \\
\text { degree }\end{array}$ & 2.30 & 76.60 & 51 & 30 & 107 & All $=188$ & & \\
\hline
\end{tabular}

From table (12) there are differences between the research groups responses to all statements except for statement number (37/1) as chi ranged between $\left(9.86^{*}: 15.22^{*}\right)$ with an outweighed percentage (75.53: 83.33).

The results of Salma Kunde (2018) (38) study indicate that the sports field has benefited greatly from information technology, represented by the emergence of new techniques to explain sports movements, the development of presentation methods, and the increase in the use of computers in reaching modern methods that enable a successfully manage of sport activity.

Studies of Salma Konda (2018) (38), Abla Zaian (2016) (3) indicates that sports field profited from information technology via emergence of new techniques of illustrating sports activities and developing display methods.

Faisal EImala (2019) mentions that international trials proved the ability of artificial intelligence to develop and improve sports performance level via analyzing as most great data as possible of players and teams. (69)

The researcher believes that it is necessary to activate the digital transformation system by introducing artificial intelligence applications within sports clubs by developing a future plan to electronically transfer all the services provided by the club, thus achieving a competitive advantage for sports clubs. 
Table (13) First Factor Results: Digital Transformation Requirements to Achieve the Best Competitive Advantage at Clubs (Third Dimension: Digital Techniques) (F- Robotic Process Automation)

\begin{tabular}{|c|c|c|c|c|c|c|c|c|c|}
\hline $\begin{array}{c}\text { Chi } \\
\text { square }\end{array}$ & Attitude & Average & $\begin{array}{c}\text { Out } \\
\text { weighted } \\
\text { percentage }\end{array}$ & Disagreeing & $\begin{array}{c}\text { To a } \\
\text { certain } \\
\text { degree }\end{array}$ & Agreeing & Departments & Statement content & $\mathbf{S}$ \\
\hline \multicolumn{9}{|c|}{ The club uses robot operations to add functional advantages represented in } & 28 \\
\hline \multirow{3}{*}{8.93} & agreeing & 2.71 & 90.36 & 9 & 6 & 68 & $\begin{array}{c}\text { Supreme } \\
\text { management }=83\end{array}$ & \multirow{4}{*}{$\begin{array}{c}\text { Managing payrolls and } \\
\text { compensations } \\
\text { registration }\end{array}$} & \multirow{4}{*}{$28 / 1$} \\
\hline & $\begin{array}{c}\text { to a } \\
\text { certain } \\
\text { degree }\end{array}$ & 2.13 & 70.83 & 6 & 2 & 8 & $\begin{array}{c}\text { Middle } \\
\text { management }=16\end{array}$ & & \\
\hline & agreeing & 2.48 & 82.77 & 19 & 8 & 62 & $\begin{array}{c}\text { Executive } \\
\text { management }=89\end{array}$ & & \\
\hline 138.43 & agreeing & 2.55 & 85.11 & 34 & 16 & 138 & All $=188$ & & \\
\hline \multirow{3}{*}{6.07} & agreeing & 2.64 & 87.95 & 11 & 8 & 64 & $\begin{array}{c}\text { Supreme } \\
\text { management }=83\end{array}$ & \multirow{4}{*}{$\begin{array}{l}\text { Managing the club's } \\
\text { employees data }\end{array}$} & \multirow{4}{*}{$28 / 2$} \\
\hline & $\begin{array}{c}\text { to a } \\
\text { certain } \\
\text { degree }\end{array}$ & 2.19 & 72.92 & 5 & 3 & 8 & $\begin{array}{c}\text { Middle } \\
\text { management }=16\end{array}$ & & \\
\hline & agreeing & 2.43 & 80.90 & 20 & 11 & 58 & $\begin{array}{c}\text { Executive } \\
\text { management }=89\end{array}$ & & \\
\hline 110.09 & agreeing & 2.50 & 83.33 & 36 & 22 & 130 & All $=188$ & & \\
\hline \multirow{3}{*}{$13.20 *$} & agreeing & 2.66 & 88.76 & 10 & 8 & 65 & $\begin{array}{c}\text { Supreme } \\
\text { management }=83\end{array}$ & \multirow{4}{*}{$\begin{array}{l}\text { Identifying credit and } \\
\text { debit accounts }\end{array}$} & \multirow{4}{*}{$28 / 3$} \\
\hline & $\begin{array}{c}\text { to a } \\
\text { certain } \\
\text { degree }\end{array}$ & 2.06 & 68.75 & 6 & 3 & 7 & $\begin{array}{c}\text { Middle } \\
\text { management }=16\end{array}$ & & \\
\hline & $\begin{array}{c}\text { to a } \\
\text { certain } \\
\text { degree }\end{array}$ & 2.31 & 77.15 & 22 & 17 & 50 & $\begin{array}{c}\text { Executive } \\
\text { management= } 89\end{array}$ & & \\
\hline 85.06 & agreeing & 2.45 & 81.56 & 38 & 28 & 122 & All $=188$ & & \\
\hline \multirow{3}{*}{$16.08 *$} & agreeing & 2.61 & 87.15 & 11 & 10 & 62 & $\begin{array}{c}\text { Supreme } \\
\text { management }=83\end{array}$ & \multirow{4}{*}{$\begin{array}{l}\text { Looking into the } \\
\text { members' complaints }\end{array}$} & \multirow{4}{*}{$28 / 4$} \\
\hline & $\begin{array}{c}\text { to a } \\
\text { certain } \\
\text { degree } \\
\end{array}$ & 1.88 & 62.50 & 7 & 4 & 5 & $\begin{array}{c}\text { Middle } \\
\text { management }=16\end{array}$ & & \\
\hline & $\begin{array}{c}\text { to a } \\
\text { certain } \\
\text { degree }\end{array}$ & 2.25 & 74.91 & 24 & 19 & 46 & $\begin{array}{c}\text { Executive } \\
\text { management= } 89\end{array}$ & & \\
\hline 61.29 & agreeing & 2.38 & 79.26 & 42 & 33 & 113 & $\mathrm{All}=\mathbf{1 8 8}$ & & \\
\hline \multirow{3}{*}{$13.59 *$} & agreeing & 2.69 & 89.56 & 9 & 8 & 66 & $\begin{array}{c}\text { Supreme } \\
\text { management }=83\end{array}$ & \multirow{4}{*}{$\begin{array}{l}\text { Managing the club's } \\
\text { members subscription } \\
\text { renewal }\end{array}$} & \multirow{4}{*}{$28 / 5$} \\
\hline & $\begin{array}{c}\text { to a } \\
\text { certain } \\
\text { degree }\end{array}$ & 2.06 & 68.75 & 7 & 1 & 8 & $\begin{array}{c}\text { Middle } \\
\text { management }=16\end{array}$ & & \\
\hline & agreeing & 2.37 & 79.03 & 21 & 14 & 54 & $\begin{array}{c}\text { Executive } \\
\text { management= 89 } \\
\end{array}$ & & \\
\hline 103.73 & agreeing & 2.48 & 82.80 & 37 & 23 & 128 & All $=188$ & & \\
\hline
\end{tabular}

From table (13) and the figure there are differences between the research groups' responses to statements numbers $(28 / 3,28 / 4,28 / 5)$ as chi square ranged between $\left(13.20^{*}: 16.08^{*}\right)$ and outweighed percentage ranged between (79.26: 82.80).

Also, the research sample opinion agreed on statements numbers $(\mathbf{2 8 / 1}, \mathbf{2 8 / 2})$ as chi square reached (8.93), (6.07) with an outweighed percentage (85.11), (83.33) respectively.

Mohit Sharma (2018) states that robotic process automation (RPA) solutions are typically 
implemented in organizations that typically depend on human resources extensively for largescale, interactive, and repetitive operations. The key processes best suited to robotic process automation tend to be extensively based on data entry, comparisons and validation. (55:10).

Abdelarahman Elour (2018) stated that to guarantee successful execution of robot operations automation through profiting from automatic control increase, the organizations shall identify and redesign their current operations. (1:4)

The researcher believes that additional robotic process automations can be used within sports clubs to conduct sensitive operations without human intervention at small costs, and to restructure human resources with work requirements, including evaluation work and rewards for workers in the affected areas.

Table (14) Second Factor Results: Digital Transformation Requirements to Achieve the Best Competitive Advantage at Clubs

(First Dimension: E. Commerce)

\begin{tabular}{|c|c|c|c|c|c|c|c|c|c|}
\hline $\begin{array}{c}\text { Chi } \\
\text { square }\end{array}$ & Attitude & Average & $\begin{array}{c}\text { Out } \\
\text { weighted } \\
\text { percentage }\end{array}$ & Disagreeing & $\begin{array}{c}\text { To a } \\
\text { certain } \\
\text { degree }\end{array}$ & Agreeing & Departments & Statement content & $\mathbf{S}$ \\
\hline & & & & & & & & $\begin{array}{l}\text { Using ecommerce by the } \\
\text { club plays a great role in }\end{array}$ & 29 \\
\hline \multirow{3}{*}{$10.83 *$} & agreeing & 2.70 & 89.96 & 8 & 9 & 66 & $\begin{array}{c}\text { Supreme } \\
\text { management }=83\end{array}$ & \multirow{4}{*}{$\begin{array}{c}\text { Achieving a competitive } \\
\text { advantage for } \\
\text { beneficiaries service }\end{array}$} & \multirow{4}{*}{$29 / 1$} \\
\hline & agreeing & 2.38 & 79.17 & 4 & 2 & 10 & $\begin{array}{c}\text { Middle } \\
\text { management }=16\end{array}$ & & \\
\hline & agreeing & 2.45 & 81.65 & 13 & 23 & 53 & $\begin{array}{c}\text { Executive } \\
\text { management }=89\end{array}$ & & \\
\hline 105.97 & agreeing & 2.55 & 85.11 & 25 & 34 & 129 & $A l l=188$ & & \\
\hline \multirow{3}{*}{$17.87 *$} & agreeing & 2.69 & 89.56 & 8 & 10 & 65 & $\begin{array}{c}\text { Supreme } \\
\text { management }=83\end{array}$ & \multirow{4}{*}{$\begin{array}{c}\text { Facilitating commercial } \\
\text { deals }\end{array}$} & \multirow{4}{*}{$29 / 2$} \\
\hline & $\begin{array}{c}\text { to a certain } \\
\text { degree }\end{array}$ & 2.13 & 70.83 & 6 & 2 & 8 & $\begin{array}{c}\text { Middle } \\
\text { management }=16\end{array}$ & & \\
\hline & agreeing & 2.44 & 81.27 & 12 & 26 & 51 & $\begin{array}{c}\text { Executive } \\
\text { management }=89\end{array}$ & & \\
\hline 91.19 & agreeing & 2.52 & 84.04 & 26 & 38 & 124 & All $=188$ & & \\
\hline \multirow{3}{*}{$12.82 *$} & agreeing & 2.63 & 87.55 & 10 & 11 & 62 & $\begin{array}{c}\text { Supreme } \\
\text { management }=83\end{array}$ & \multirow{4}{*}{$\begin{array}{l}\text { Reducing the club's } \\
\text { expenses }\end{array}$} & \multirow{4}{*}{$29 / 3$} \\
\hline & $\begin{array}{c}\text { to a certain } \\
\text { degree }\end{array}$ & 1.94 & 64.58 & 6 & 5 & 5 & $\begin{array}{c}\text { Middle } \\
\text { management }=16\end{array}$ & & \\
\hline & agreeing & 2.44 & 81.27 & 15 & 20 & 54 & $\begin{array}{c}\text { Executive } \\
\text { management }=89\end{array}$ & & \\
\hline 81.65 & agreeing & 2.48 & 82.62 & 31 & 36 & 121 & $A l l=188$ & & \\
\hline \multirow{3}{*}{$13.94 *$} & agreeing & 2.48 & 82.73 & 16 & 11 & 56 & $\begin{array}{c}\text { Supreme } \\
\text { management }=83\end{array}$ & \multirow{4}{*}{$\begin{array}{l}\text { The club provides e. } \\
\text { payment service to } \\
\text { facilitate payment and } \\
\text { reducing cash collection } \\
\text { corruption }\end{array}$} & \multirow{4}{*}{30} \\
\hline & $\begin{array}{c}\text { to a certain } \\
\text { degree }\end{array}$ & 1.69 & 56.25 & 8 & 5 & 3 & $\begin{array}{c}\text { Middle } \\
\text { management }=16\end{array}$ & & \\
\hline & agreeing & 2.37 & 79.03 & 19 & 18 & 52 & $\begin{array}{c}\text { Executive } \\
\text { management }=89\end{array}$ & & \\
\hline 56.56 & agreeing & 2.36 & 78.72 & 43 & 34 & 111 & $A l l=188$ & & \\
\hline \multirow{2}{*}{$13.75 *$} & agreeing & 2.64 & 87.95 & 10 & 10 & 63 & $\begin{array}{c}\text { Supreme } \\
\text { management }=83\end{array}$ & \multirow{2}{*}{$\begin{array}{c}\text { There is an attitude } \\
\text { towards mechanizing } \\
\text { joining championships } \\
\text { and academies to }\end{array}$} & \multirow{2}{*}{31} \\
\hline & $\begin{array}{c}\text { to a certain } \\
\text { degree }\end{array}$ & 1.88 & 62.50 & 7 & 4 & 5 & $\begin{array}{c}\text { Middle } \\
\text { management }=16\end{array}$ & & \\
\hline
\end{tabular}




\begin{tabular}{|c|c|c|c|c|c|c|c|c|c|}
\hline $\begin{array}{l}\text { Chi } \\
\text { square }\end{array}$ & Attitude & Average & $\begin{array}{c}\text { Out } \\
\text { weighted } \\
\text { percentage }\end{array}$ & Disagreeing & $\begin{array}{l}\text { To a } \\
\text { certain } \\
\text { degree }\end{array}$ & Agreeing & Departments & Statement content & $\mathbf{S}$ \\
\hline & agreeing & 2.42 & 80.52 & 18 & 16 & 55 & $\begin{array}{c}\text { Executive } \\
\text { management }=89\end{array}$ & \multirow{2}{*}{$\begin{array}{l}\text { facilitate counting } \\
\text { practices and creating } \\
\text { good data bases }\end{array}$} & \\
\hline 87.33 & agreeing & 2.47 & 82.27 & 35 & 30 & 123 & All $=188$ & & \\
\hline \multirow{3}{*}{$14.71 *$} & $\overline{\text { agreeing }}$ & 2.59 & 86.35 & 11 & 12 & 60 & $\begin{array}{c}\text { Supreme } \\
\text { management }=83\end{array}$ & \multirow{4}{*}{$\begin{array}{l}\text { ts } \\
\text { ins } \\
\text { er }\end{array}$} & \multirow{4}{*}{32} \\
\hline & $\begin{array}{c}\text { to a certain } \\
\text { degree }\end{array}$ & 1.88 & 62.50 & 6 & 6 & 4 & $\begin{array}{c}\text { Middle } \\
\text { management }=16\end{array}$ & & \\
\hline & $\begin{array}{c}\text { to a certain } \\
\text { degree }\end{array}$ & 2.31 & 77.15 & 20 & 21 & 48 & $\begin{array}{c}\text { Executive } \\
\text { management }=89\end{array}$ & & \\
\hline 58.29 & agreeing & 2.40 & 79.96 & 37 & 39 & 112 & All $=188$ & & \\
\hline \multirow{3}{*}{$14.27 *$} & agreeing & 2.59 & 86.35 & 13 & 8 & 62 & $\begin{array}{c}\text { Supreme } \\
\text { management }=83\end{array}$ & \multirow{4}{*}{$\begin{array}{l}\text { Creating a digital data } \\
\text { base according to which } \\
\text { organizations, their place } \\
\text { and types are selected }\end{array}$} & \multirow{4}{*}{33} \\
\hline & $\begin{array}{c}\text { to a certain } \\
\text { degree }\end{array}$ & 2.06 & 68.75 & 5 & 5 & 6 & $\begin{array}{c}\text { Middle } \\
\text { management }=16\end{array}$ & & \\
\hline & $\begin{array}{c}\text { to a certain } \\
\text { degree }\end{array}$ & 2.28 & 76.03 & 21 & 22 & 46 & $\begin{array}{c}\text { Executive } \\
\text { management }=89\end{array}$ & & \\
\hline 63.20 & agreeing & 2.40 & 79.96 & 39 & 35 & 114 & All $=188$ & & \\
\hline \multirow{3}{*}{$11.75 *$} & agreeing & 2.46 & 81.93 & 17 & 11 & 55 & $\begin{array}{c}\text { Supreme } \\
\text { management }=83\end{array}$ & \multirow{4}{*}{$\begin{array}{l}\text { E. control via system } \\
\text { disallowing registration } \\
\text { unless legally } \\
\text { accordingly pinpointing } \\
\text { and dealing with } \\
\text { violations }\end{array}$} & \multirow{4}{*}{34} \\
\hline & $\begin{array}{l}\text { to a certain } \\
\text { degree }\end{array}$ & 1.88 & 62.50 & 7 & 4 & 5 & $\begin{array}{c}\text { Middle } \\
\text { management }=16\end{array}$ & & \\
\hline & $\begin{array}{c}\text { to a certain } \\
\text { degree }\end{array}$ & 2.27 & 75.66 & 20 & 25 & 44 & $\begin{array}{c}\text { Executive } \\
\text { management= } 89\end{array}$ & & \\
\hline \multirow[t]{2}{*}{41.02} & $\begin{array}{l}\text { to a certain } \\
\text { degree }\end{array}$ & 2.32 & $\mathbf{7 7 . 3 0}$ & 44 & 40 & 104 & All $=188$ & & \\
\hline & & & & & & & & $\begin{array}{l}\text { Digital transformation } \\
\text { supports the club } \\
\text { administrative review } \\
\text { via: }\end{array}$ & 35 \\
\hline \multirow{3}{*}{7.13} & agreeing & 2.71 & 90.39 & 8 & 8 & 67 & $\begin{array}{c}\text { Supreme } \\
\text { management }=83\end{array}$ & \multirow{4}{*}{$\begin{array}{c}\text { Rationalizing } \\
\text { administrative decisions }\end{array}$} & \multirow{4}{*}{$35 / 1$} \\
\hline & $\begin{array}{c}\text { to a certain } \\
\text { degree }\end{array}$ & 2.31 & 77.08 & 4 & 3 & 9 & $\begin{array}{c}\text { Middle } \\
\text { management= } 16\end{array}$ & & \\
\hline & agreeing & 2.46 & 82.02 & 17 & 14 & 58 & $\begin{array}{c}\text { Executive } \\
\text { management }=89\end{array}$ & & \\
\hline 121.93 & agreeing & 2.56 & 85.25 & 29 & 25 & 134 & All $=188$ & & \\
\hline \multirow{3}{*}{$12.30 *$} & agreeing & 2.71 & 90.36 & 8 & 8 & 67 & $\begin{array}{c}\text { Supreme } \\
\text { management }=83\end{array}$ & \multirow{4}{*}{$\begin{array}{l}\text { Supporting internal } \\
\text { control system }\end{array}$} & \multirow{4}{*}{$35 / 2$} \\
\hline & $\begin{array}{c}\text { to a certain } \\
\text { degree }\end{array}$ & 2.25 & 75.00 & 4 & 4 & 8 & $\begin{array}{c}\text { Middle } \\
\text { management }=16\end{array}$ & & \\
\hline & agreeing & 2.36 & 78.65 & 20 & 17 & 52 & $\begin{array}{c}\text { Executive } \\
\text { management= } 89\end{array}$ & & \\
\hline 99.14 & agreeing & 2.51 & 83.51 & 32 & 29 & 127 & All $=188$ & & \\
\hline \multirow{3}{*}{$12.22 *$} & agreeing & 2.69 & 89.56 & 8 & 10 & 65 & $\begin{array}{c}\text { Supreme } \\
\text { management }=83\end{array}$ & \multirow{4}{*}{$\begin{array}{c}\text { Verifying observing the } \\
\text { planned administrative } \\
\text { policies }\end{array}$} & \multirow{4}{*}{$35 / 3$} \\
\hline & $\begin{array}{l}\text { to a certain } \\
\text { degree }\end{array}$ & 2.19 & 72.92 & 4 & 5 & 7 & $\begin{array}{c}\text { Middle } \\
\text { management }=16\end{array}$ & & \\
\hline & agreeing & 2.36 & 78.65 & 19 & 19 & 51 & $\begin{array}{c}\text { Executive } \\
\text { management= } 89\end{array}$ & & \\
\hline 87.20 & agreeing & 2.49 & 82.98 & 31 & 34 & 123 & All $=188$ & & \\
\hline \multirow{3}{*}{$10.31 *$} & agreeing & 2.70 & 89.96 & 8 & 9 & 66 & $\begin{array}{c}\text { Supreme } \\
\text { management }=83\end{array}$ & \multirow{3}{*}{$\begin{array}{l}\text { Verifying the financial } \\
\text { reports true information }\end{array}$} & \multirow{3}{*}{$35 / 4$} \\
\hline & $\begin{array}{c}\text { to a certain } \\
\text { degree }\end{array}$ & 2.25 & 75.00 & 4 & 4 & 8 & $\begin{array}{c}\text { Middle } \\
\text { management }=16\end{array}$ & & \\
\hline & agreeing & 2.93 & 79.78 & 18 & 18 & 53 & $\begin{array}{c}\text { Executive } \\
\text { management }=89\end{array}$ & & \\
\hline
\end{tabular}




\begin{tabular}{|c|c|c|c|c|c|c|c|c|c|}
\hline $\begin{array}{l}\text { Chi } \\
\text { square }\end{array}$ & Attitude & Average & $\begin{array}{c}\text { Out } \\
\text { weighted } \\
\text { percentage }\end{array}$ & Disagreeing & $\begin{array}{c}\text { To a } \\
\text { certain } \\
\text { degree }\end{array}$ & Agreeing & Departments & Statement content & $\mathbf{S}$ \\
\hline 99.07 & agreeing & 2.52 & 83.87 & 30 & 31 & 127 & All $=188$ & & \\
\hline \multirow{3}{*}{$12.24 *$} & agreeing & 2.63 & 87.55 & 10 & 11 & 62 & $\begin{array}{c}\text { Supreme } \\
\text { management }=83\end{array}$ & \multirow{4}{*}{$\begin{array}{l}\text { Evaluating the club' s } \\
\text { employees performance }\end{array}$} & \multirow{4}{*}{$35 / 5$} \\
\hline & sometimes & 2.00 & 66.67 & 6 & 4 & 6 & $\begin{array}{c}\text { Middle } \\
\text { management }=16\end{array}$ & & \\
\hline & sometimes & 2.33 & $\mathbf{7 7 . 5 3}$ & 20 & 20 & 49 & $\begin{array}{c}\text { Executive } \\
\text { management }=89\end{array}$ & & \\
\hline \multirow[t]{2}{*}{70.67} & agreeing & 2.43 & 81.03 & 36 & 35 & 117 & All $=188$ & & \\
\hline & & & & & & & & $\begin{array}{l}\text { E. accounting systems } \\
\text { suitable data about }\end{array}$ & 36 \\
\hline \multirow{3}{*}{$11.82 *$} & agreeing & 2.67 & 89.16 & 9 & 9 & 65 & $\begin{array}{c}\text { Supreme } \\
\text { management }=83\end{array}$ & \multirow{4}{*}{ The net club's profit } & \multirow{4}{*}{$36 / 1$} \\
\hline & $\begin{array}{c}\text { to a certain } \\
\text { degree }\end{array}$ & 2.06 & 68.75 & 5 & 5 & 6 & $\begin{array}{c}\text { Middle } \\
\text { management }=16 \\
\end{array}$ & & \\
\hline & agreeing & 2.44 & 81.27 & 18 & 14 & 57 & $\begin{array}{c}\text { Executive } \\
\text { management }=89\end{array}$ & & \\
\hline 102.30 & agreeing & 2.51 & 83.69 & 32 & 28 & 128 & All $=188$ & & \\
\hline \multirow{3}{*}{$13.54 *$} & agreeing & 2.69 & 89.56 & 9 & 8 & 66 & $\begin{array}{c}\text { Supreme } \\
\text { management }=83\end{array}$ & \multirow{4}{*}{$\begin{array}{l}\text { Average investment } \\
\text { return }\end{array}$} & \multirow{4}{*}{$36 / 2$} \\
\hline & $\begin{array}{c}\text { to a certain } \\
\text { degree }\end{array}$ & 2.00 & 66.67 & 6 & 4 & 6 & $\begin{array}{c}\text { Middle } \\
\text { management }=16\end{array}$ & & \\
\hline & agreeing & 2.40 & 80.15 & 19 & 15 & 55 & $\begin{array}{c}\text { Executive } \\
\text { management }=89\end{array}$ & & \\
\hline 99.46 & agreeing & 2.49 & 83.16 & 34 & 27 & 127 & All $=188$ & & \\
\hline \multirow{3}{*}{$14.57 *$} & agreeing & 2.65 & 88.35 & 10 & 9 & 64 & $\begin{array}{c}\text { Supreme } \\
\text { management }=83\end{array}$ & \multirow{4}{*}{$\begin{array}{c}\text { Declared liquid money } \\
\text { percentage }\end{array}$} & \multirow{4}{*}{$36 / 3$} \\
\hline & $\begin{array}{c}\text { to a certain } \\
\text { degree }\end{array}$ & 1.88 & 62.50 & 7 & 4 & 5 & $\begin{array}{c}\text { Middle } \\
\text { management }=16 \\
\end{array}$ & & \\
\hline & agreeing & 2.44 & 81.27 & 17 & 16 & 56 & $\begin{array}{c}\text { Executive } \\
\text { management }=89\end{array}$ & & \\
\hline 93.20 & agreeing & 2.48 & 82.80 & 34 & 29 & 125 & All $=188$ & & \\
\hline
\end{tabular}

From table (14) there are significant differences the research sample groups' responses for all the dimensions statements as chi square ranged between $(10.31 *: 17.87 *)$ and outweighed percentage ranged between $(77.30: 85.11)$, except for statement number $(35 / 1)$ as chi square reached (7.13).

Results of study of Omar Khalaf (2019) (32), Ahmed Adam (2014) (5) indicate that using information technology in administrative business is the aim of all organization to achieve the competitive advantage.

Nigm Aboud Nigm (2004) states e. control advantages, as permanent control is achieved instead of periodic control; and by time, reduces the importance of control depending on inputs, processes or activities in the interest of increasing results assurance $(31: 247)$.

The researcher concludes that the shift towards electronic commerce has become a necessary and vital matter, and therefore club administrations should bear this in mind in setting the club's marketing strategies, in order to reduce advertising and advertising costs, and facilitate the beneficiaries' acquaintance with the specifications of the services provided by the club, thus 
achieving a competitive advantage among different sports clubs.

\section{Table (15) Second Factor Results: Digital Transformation Requirements to Achieve the Best Competitive Advantage at Clubs}

(Second Dimension: E. Marketing) (A- The Club' Website)

\begin{tabular}{|c|c|c|c|c|c|c|c|c|c|}
\hline $\begin{array}{c}\text { Chi } \\
\text { square }\end{array}$ & Attitude & Average & $\begin{array}{c}\text { Out } \\
\text { weighted } \\
\text { percentage }\end{array}$ & Disagreeing & $\begin{array}{c}\text { To a } \\
\text { certain } \\
\text { degree }\end{array}$ & Agreeing & Departments & Statement content & $\mathbf{S}$ \\
\hline \multirow{3}{*}{4.31} & agreeing & 2.69 & 89.56 & 8 & 10 & 65 & $\begin{array}{c}\text { Supreme } \\
\text { management }=83\end{array}$ & \multirow{4}{*}{$\begin{array}{l}\text { The club has a website } \\
\text { in Arabic and English } \\
\text { to facilitate dealing } \\
\text { with and access to }\end{array}$} & \multirow{4}{*}{37} \\
\hline & $\begin{array}{c}\text { to a certain } \\
\text { degree }\end{array}$ & 2.31 & 77.08 & 4 & 3 & 9 & $\begin{array}{c}\text { Middle } \\
\text { management }=16\end{array}$ & & \\
\hline & agreeing & 2.55 & 85.02 & 13 & 14 & 62 & $\begin{array}{c}\text { Executive } \\
\text { management }=89\end{array}$ & & \\
\hline 128.76 & agreeing & 2.59 & 86.35 & 25 & 27 & 136 & All $=188$ & & \\
\hline \multirow{3}{*}{6.20} & agreeing & 2.71 & 90.63 & 8 & 8 & 67 & $\begin{array}{c}\text { Supreme } \\
\text { management }=\mathbf{8 3}\end{array}$ & \multirow{4}{*}{$\begin{array}{c}\text { The club's website is } \\
\text { characterized by easy } \\
\text { use }\end{array}$} & \multirow{4}{*}{38} \\
\hline & $\begin{array}{l}\text { to a certain } \\
\text { degree }\end{array}$ & 2.31 & 77.08 & 4 & 3 & 9 & $\begin{array}{c}\text { Middle } \\
\text { management }=16\end{array}$ & & \\
\hline & agreeing & 2.51 & 83.52 & 15 & 14 & 60 & $\begin{array}{c}\text { Executive } \\
\text { management }=89\end{array}$ & & \\
\hline 128.76 & agreeing & 2.58 & 85.99 & 27 & 25 & 136 & All $=188$ & & \\
\hline \multirow{3}{*}{6.47} & agreeing & 2.65 & 88.35 & 8 & 13 & 62 & $\begin{array}{c}\text { Supreme } \\
\text { management }=83\end{array}$ & \multirow{4}{*}{$\begin{array}{c}\text { The club's page } \\
\text { includes a description } \\
\text { of services and } \\
\text { activities provided with } \\
\text { pictures }\end{array}$} & \multirow{4}{*}{39} \\
\hline & $\begin{array}{c}\text { to a certain } \\
\text { degree }\end{array}$ & 2.19 & 72.92 & 5 & 3 & 8 & $\begin{array}{c}\text { Middle } \\
\text { management }=16\end{array}$ & & \\
\hline & agreeing & 2.46 & 82.02 & 17 & 14 & 58 & $\begin{array}{c}\text { Executive } \\
\text { management }=89\end{array}$ & & \\
\hline 102.17 & agreeing & 2.52 & 84.04 & 30 & 30 & 128 & All $=188$ & & \\
\hline \multirow{3}{*}{9.17} & agreeing & 2.66 & 88.76 & 8 & 12 & 63 & $\begin{array}{c}\text { Supreme } \\
\text { management }=83\end{array}$ & \multirow{4}{*}{$\begin{array}{c}\text { The club tops the first } \\
\text { search engine pages }\end{array}$} & \multirow{4}{*}{40} \\
\hline & $\begin{array}{l}\text { to a certain } \\
\text { degree }\end{array}$ & 2.06 & 68.75 & 6 & 3 & 7 & $\begin{array}{c}\text { Middle } \\
\text { management }=16\end{array}$ & & \\
\hline & agreeing & 2.52 & 83.90 & 15 & 13 & 61 & $\begin{array}{c}\text { Executive } \\
\text { management }=89\end{array}$ & & \\
\hline 11.78 & agreeing & 2.54 & 84.75 & 29 & 28 & 131 & All $=188$ & & \\
\hline \multirow{3}{*}{$12.30 *$} & agreeing & 2.66 & 88.76 & 9 & 10 & 64 & $\begin{array}{c}\text { Supreme } \\
\text { management }=83\end{array}$ & \multirow{4}{*}{$\begin{array}{c}\text { The club updates } \\
\text { information } \\
\text { periodically on website }\end{array}$} & \multirow{4}{*}{41} \\
\hline & $\begin{array}{c}\text { to a certain } \\
\text { degree }\end{array}$ & 2.00 & 66.67 & 7 & 2 & 7 & $\begin{array}{c}\text { Middle } \\
\text { management }=16\end{array}$ & & \\
\hline & agreeing & 2.46 & 82.02 & 16 & 16 & 57 & $\begin{array}{c}\text { Executive } \\
\text { management }=89\end{array}$ & & \\
\hline 102.30 & agreeing & 2.51 & 83.69 & 32 & 28 & 128 & All $=188$ & & \\
\hline \multirow{3}{*}{$11.55 *$} & agreeing & 2.70 & 89.96 & 8 & 9 & 66 & $\begin{array}{c}\text { Supreme } \\
\text { management }=\mathbf{8 3}\end{array}$ & \multirow{4}{*}{$\begin{array}{l}\text { Words used on the } \\
\text { club's website are } \\
\text { studied carefully by a } \\
\text { specialized department }\end{array}$} & \multirow{4}{*}{42} \\
\hline & $\begin{array}{c}\text { to a certain } \\
\text { degree }\end{array}$ & 2.13 & 70.83 & 6 & 2 & 8 & $\begin{array}{c}\text { Middle } \\
\text { management }=16\end{array}$ & & \\
\hline & agreeing & 2.42 & 80.25 & 18 & 16 & 55 & $\begin{array}{c}\text { Executive } \\
\text { management }=89\end{array}$ & & \\
\hline 105.52 & agreeing & 2.52 & 83.87 & 32 & 27 & 129 & All $=188$ & & \\
\hline \multirow{3}{*}{$14.14 *$} & agreeing & 2.69 & 89.56 & 8 & 10 & 65 & $\begin{array}{c}\text { Supreme } \\
\text { management }=83\end{array}$ & \multirow{4}{*}{$\begin{array}{l}\text { The club has many } \\
\text { specialists of } \\
\text { developing the club's } \\
\text { website }\end{array}$} & \multirow{4}{*}{43} \\
\hline & $\begin{array}{c}\text { to a certain } \\
\text { degree }\end{array}$ & 1.94 & 64.58 & 7 & 3 & 6 & $\begin{array}{c}\text { Middle } \\
\text { management }=16\end{array}$ & & \\
\hline & agreeing & 2.49 & 83.15 & 15 & 15 & 59 & $\begin{array}{c}\text { Executive } \\
\text { management }=89\end{array}$ & & \\
\hline 108.55 & agreeing & 2.53 & 84.40 & 30 & 28 & 130 & All $=188$ & & \\
\hline
\end{tabular}




\begin{tabular}{|c|c|c|c|c|c|c|c|c|c|}
\hline $\begin{array}{l}\text { Chi } \\
\text { square }\end{array}$ & Attitude & Average & $\begin{array}{c}\text { Out } \\
\text { weighted } \\
\text { percentage }\end{array}$ & Disagreeing & $\begin{array}{c}\text { To a } \\
\text { certain } \\
\text { degree }\end{array}$ & Agreeing & Departments & Statement content & $\mathbf{S}$ \\
\hline \multirow{3}{*}{$19.78 *$} & agreeing & 2.63 & 87.55 & 10 & 11 & 62 & $\begin{array}{c}\text { Supreme } \\
\text { management }=83\end{array}$ & \multirow{4}{*}{$\begin{array}{c}\text { There are financial } \\
\text { appropriations } \\
\text { allocated for searching } \\
\text { and developing the } \\
\text { website }\end{array}$} & \multirow{4}{*}{44} \\
\hline & $\begin{array}{c}\text { to a certain } \\
\text { degree }\end{array}$ & 1.75 & 58.33 & 9 & 2 & 5 & $\begin{array}{c}\text { Middle } \\
\text { management }=16\end{array}$ & & \\
\hline & agreeing & 2.36 & 78.65 & 19 & 19 & 51 & $\begin{array}{c}\text { Executive } \\
\text { management }=89\end{array}$ & & \\
\hline 73.57 & agreeing & 2.43 & 80.85 & 38 & 32 & 118 & All $=188$ & & \\
\hline \multirow{3}{*}{$15.49 *$} & agreeing & 2.66 & 88.76 & 8 & 12 & 63 & $\begin{array}{c}\text { Supreme } \\
\text { management }=83\end{array}$ & \multirow{4}{*}{$\begin{array}{l}\text { There is an icon of } \\
\text { direct communication } \\
\text { via the website to } \\
\text { provide the direct } \\
\text { support to the club's } \\
\text { beneficiaries }\end{array}$} & \multirow{4}{*}{45} \\
\hline & $\begin{array}{c}\text { to a certain } \\
\text { degree }\end{array}$ & 1.75 & 58.33 & 7 & 3 & 5 & $\begin{array}{c}\text { Middle } \\
\text { management }=16\end{array}$ & & \\
\hline & agreeing & 2.40 & 80.15 & 17 & 19 & 53 & $\begin{array}{c}\text { Executive } \\
\text { management }=89\end{array}$ & & \\
\hline 82.42 & agreeing & 2.46 & 82.09 & 32 & 34 & 121 & All $=188$ & & \\
\hline \multirow{3}{*}{$17.60 *$} & agreeing & 2.54 & 84.74 & 11 & 16 & 56 & $\begin{array}{c}\text { Supreme } \\
\text { management }=83\end{array}$ & \multirow{4}{*}{$\begin{array}{c}\text { Enabling beneficiaries } \\
\text { to update their data } \\
\text { electronically via the } \\
\text { club's website }\end{array}$} & \multirow{4}{*}{46} \\
\hline & $\begin{array}{c}\begin{array}{c}\text { to a certain } \\
\text { degree }\end{array} \\
\end{array}$ & 1.69 & 56.25 & 9 & 3 & 4 & $\begin{array}{c}\text { Middle } \\
\text { management }=16\end{array}$ & & \\
\hline & agreeing & 2.40 & 80.15 & 16 & 21 & 52 & $\begin{array}{c}\text { Executive } \\
\text { management }=89\end{array}$ & & \\
\hline 58.38 & agreeing & 2.40 & 80.14 & 36 & 40 & 112 & All $=188$ & & \\
\hline \multirow{3}{*}{$15.94 *$} & agreeing & 2.66 & 88.76 & 9 & 10 & 64 & $\begin{array}{c}\text { Supreme } \\
\text { management }=83\end{array}$ & \multirow{4}{*}{$\begin{array}{l}\text { The club focuses on } \\
\text { preparing the page } \\
\text { followers, number of } \\
\text { visits and causes of } \\
\text { their increase or } \\
\text { decrease }\end{array}$} & \multirow{4}{*}{47} \\
\hline & $\begin{array}{c}\text { to a certain } \\
\text { degree }\end{array}$ & 1.81 & 60.42 & 8 & 3 & 5 & $\begin{array}{c}\text { Middle } \\
\text { management }=16\end{array}$ & & \\
\hline & agreeing & 2.46 & 82.02 & 19 & 10 & 60 & $\begin{array}{c}\text { Executive } \\
\text { management }=89\end{array}$ & & \\
\hline 106.67 & agreeing & 2.49 & 83.16 & 36 & 23 & 129 & All $=188$ & & \\
\hline \multirow{3}{*}{$17.19 *$} & agreeing & 2.58 & 85.94 & 11 & 13 & 59 & $\begin{array}{c}\text { Supreme } \\
\text { management }=83\end{array}$ & \multirow{4}{*}{$\begin{array}{l}\text { Matches tickets are } \\
\text { offered on the club's } \\
\text { website in conformity } \\
\text { with the event } \\
\text { importance and } \\
\text { preparing the expected } \\
\text { visitors }\end{array}$} & \multirow{4}{*}{48} \\
\hline & $\begin{array}{c}\begin{array}{c}\text { to a certain } \\
\text { degree }\end{array} \\
\end{array}$ & 1.69 & 56.25 & 9 & 3 & 4 & $\begin{array}{c}\text { Middle } \\
\text { management }=16\end{array}$ & & \\
\hline & agreeing & 2.45 & 81.65 & 17 & 15 & 57 & $\begin{array}{c}\text { Executive } \\
\text { management }=89\end{array}$ & & \\
\hline 78.97 & agreeing & 2.44 & 81.38 & 37 & 31 & 120 & All $=188$ & & \\
\hline
\end{tabular}

From table (15) there are research samples groups' opinions agreement on statements numbers $(37,38,39,40)$ as chi square reached $(4.31: 9.17)$ with an outweighed percentage (84.04: 86.35).

Results of study of Zahef Mohamed (2018) (43) indicate sports organizations' keenness to availing a website and allocating an enough budget to develop e. marketing

Mohamed Ramadan (2006) clarifies that e. marketing provides the profit of investing and managing time for beneficiaries via quick searching for activities and services (28: 126).

Also from the above table, it is clear that the research sample opinions disagree on statements number (41: 48) as chi square reached $\left(11.55^{*}: 19.78^{*}\right)$ with an outweighed percentage (70.85: 84.40$)$

The results of the study of Saad Ahmed Shalaby and Abd Al-Taif Bukhara (2008) (35) indicate that most German clubs take into account the design of their web pages to ensure the management and marketing of their services to various target groups via the Internet.

Results of study of Haitham Fayez (2016) (17) proves no budget allocated for e. marketing and 
discouraging using new e. marketing means for sports activities.

The researcher concludes that the application of electronic marketing to sports clubs requires the availability of specialized human resources with the skills and qualifications that enable them to develop the club's website, with the allocation of a sufficient budget to develop its marketing activity.

Table (16) Second Factor Results: Digital Transformation Requirements to Achieve the Best Competitive Advantage at Clubs

(Second Dimension: E. Marketing) (B- the Club's E. Mail)

\begin{tabular}{|c|c|c|c|c|c|c|c|c|c|}
\hline $\begin{array}{c}\text { Chi } \\
\text { square }\end{array}$ & Attitude & Average & $\begin{array}{c}\text { Out } \\
\text { weighted } \\
\text { percentage } \\
\end{array}$ & Disagreeing & $\begin{array}{c}\text { To a } \\
\text { certain } \\
\text { degree } \\
\end{array}$ & Agreeing & Departments & Statement content & $\mathbf{S}$ \\
\hline \multirow{3}{*}{$11.11 *$} & agreeing & 2.58 & 85.94 & 12 & 11 & 60 & $\begin{array}{c}\text { Supreme } \\
\text { management }=83\end{array}$ & \multirow{4}{*}{$\begin{array}{l}\text { The club has mail } \\
\text { addresses to be used } \\
\text { in correspondences }\end{array}$} & \multirow{4}{*}{49} \\
\hline & $\begin{array}{c}\text { to a certain } \\
\text { degree }\end{array}$ & 2.00 & 66.67 & 7 & 2 & 7 & $\begin{array}{c}\text { Middle } \\
\text { management }=16\end{array}$ & & \\
\hline & $\begin{array}{l}\text { to a certain } \\
\text { degree }\end{array}$ & 2.33 & $\mathbf{7 7 . 5 3}$ & 20 & 20 & 49 & $\begin{array}{c}\text { Executive } \\
\text { management }=89\end{array}$ & & \\
\hline 68.37 & agreeing & 2.41 & 80.32 & 39 & 33 & 116 & All $=188$ & & \\
\hline \multirow{3}{*}{$12.97 *$} & agreeing & 2.54 & 84.74 & 13 & 12 & 58 & $\begin{array}{c}\text { Supreme } \\
\text { management }=83\end{array}$ & \multirow{4}{*}{$\begin{array}{c}\text { Approving the email } \\
\text { as an official method } \\
\text { of correspondences to } \\
\text { get rid of } \\
\text { documentary cycle }\end{array}$} & \multirow{4}{*}{50} \\
\hline & $\begin{array}{c}\text { to a certain } \\
\text { degree }\end{array}$ & 1.88 & 62.50 & 8 & 2 & 6 & $\begin{array}{c}\text { Middle } \\
\text { management }=16\end{array}$ & & \\
\hline & $\begin{array}{c}\text { to a certain } \\
\text { degree }\end{array}$ & 2.29 & 76.40 & 21 & 21 & 47 & $\begin{array}{c}\text { Executive } \\
\text { management }=89\end{array}$ & & \\
\hline 56.31 & agreeing & 2.37 & 78.90 & 42 & 35 & 111 & All $=188$ & & \\
\hline \multirow{3}{*}{$12.69 *$} & agreeing & 2.57 & 85.54 & 14 & 8 & 61 & $\begin{array}{c}\text { Supreme } \\
\text { management }=83\end{array}$ & \multirow{4}{*}{$\begin{array}{l}\text { The club uses the } \\
\text { email to answer the } \\
\text { beneficiaries } \\
\text { questions }\end{array}$} & \multirow{4}{*}{51} \\
\hline & $\begin{array}{l}\text { to a certain } \\
\text { degree }\end{array}$ & 1.94 & 64.58 & 7 & 3 & 6 & $\begin{array}{c}\text { Middle } \\
\text { management }=16\end{array}$ & & \\
\hline & agreeing & 2.35 & 78.28 & 19 & 20 & 50 & $\begin{array}{c}\text { Executive } \\
\text { management }=89\end{array}$ & & \\
\hline 71.31 & agreeing & 2.41 & 80.352 & 40 & 31 & 117 & All $=188$ & & \\
\hline \multirow{3}{*}{$17.56 *$} & agreeing & 2.65 & 88.35 & 9 & 11 & 63 & $\begin{array}{c}\text { Supreme } \\
\text { management }=83\end{array}$ & \multirow{4}{*}{$\begin{array}{l}\text { The beneficiaries are } \\
\text { answered quickly to } \\
\text { save time and effort }\end{array}$} & \multirow{4}{*}{52} \\
\hline & $\begin{array}{c}\text { to a certain } \\
\text { degree }\end{array}$ & 1.81 & 60.42 & 8 & 3 & 5 & $\begin{array}{c}\text { Middle } \\
\text { management }=16\end{array}$ & & \\
\hline & agreeing & 2.35 & 78.28 & 20 & 18 & 51 & $\begin{array}{c}\text { Executive } \\
\text { management }=89\end{array}$ & & \\
\hline 76.16 & agreeing & 2.44 & 81.21 & 37 & 32 & 119 & All $=188$ & & \\
\hline \multirow{3}{*}{$17.10 *$} & agreeing & 2.53 & 84.34 & 11 & 17 & 55 & $\begin{array}{c}\text { Supreme } \\
\text { management }=83\end{array}$ & \multirow{4}{*}{$\begin{array}{c}\text { There is a team } \\
\text { specialized in } \\
\text { answering the } \\
\text { beneficiaries' } \\
\text { questions via email }\end{array}$} & \multirow{4}{*}{53} \\
\hline & $\begin{array}{c}\text { to a certain } \\
\text { degree }\end{array}$ & 1.75 & 58.33 & 9 & 2 & 5 & $\begin{array}{c}\text { Middle } \\
\text { management }=16\end{array}$ & & \\
\hline & $\begin{array}{c}\text { to a certain } \\
\text { degree }\end{array}$ & 2.22 & 74.16 & 23 & 23 & 43 & $\begin{array}{c}\text { Executive } \\
\text { management }=89\end{array}$ & & \\
\hline 38.95 & $\begin{array}{c}\text { to a certain } \\
\text { degree }\end{array}$ & 2.32 & 77.30 & 43 & 42 & 103 & All $=188$ & & \\
\hline \multirow{3}{*}{$14.95 *$} & agreeing & 2.61 & 87.15 & 10 & 12 & 61 & $\begin{array}{c}\text { Supreme } \\
\text { management }=83\end{array}$ & \multirow{4}{*}{$\begin{array}{c}\text { Marketing fliers are } \\
\text { sent to the club's } \\
\text { beneficiaries via } \\
\text { email }\end{array}$} & \multirow{4}{*}{54} \\
\hline & $\begin{array}{l}\text { to a certain } \\
\text { degree }\end{array}$ & 1.94 & 64.58 & 7 & 3 & 6 & $\begin{array}{c}\text { Middle } \\
\text { management }=16\end{array}$ & & \\
\hline & $\begin{array}{c}\text { to a certain } \\
\text { degree }\end{array}$ & 2.24 & 74.53 & 24 & 20 & 45 & $\begin{array}{c}\text { Executive } \\
\text { management }=89\end{array}$ & & \\
\hline 58.54 & agreeing & 2.38 & 79.26 & 41 & 35 & 112 & All $=188$ & & \\
\hline
\end{tabular}

From table (16), there are significant differences between responses of all the research sample 
groups as chi square reached $\left(11.11^{*}: 17.56^{*}\right)$ with an outweighed percentage (77.30: 81.21$)$.

Study of Hind Elghanem (2014) (20) states that the ability of dealing with beneficiaries via email is one of the most important things introduced by knowledge and organizations digital transformation.

The researcher believes that the ability to deal with beneficiaries via e-mail and social networking services is one of the most important things that knowledge and digital transformation can provide to sports clubs in the field of receiving and responding to beneficiaries' inquiries and questions.

Table (17) Second Dimension Result: E. Marketing (C-Applications of Smart Phones and Social Media)

\begin{tabular}{|c|c|c|c|c|c|c|c|c|c|}
\hline $\begin{array}{c}\text { Chi } \\
\text { square }\end{array}$ & Attitude & Average & $\begin{array}{c}\text { Out } \\
\text { weighted } \\
\text { percentage }\end{array}$ & Disagreeing & $\begin{array}{c}\text { To a } \\
\text { certain } \\
\text { degree }\end{array}$ & Agreeing & Departments & Statement content & $\mathbf{S}$ \\
\hline \multirow{3}{*}{8.99} & agreeing & 2.49 & 83.13 & 13 & 16 & 54 & $\begin{array}{c}\text { Supreme } \\
\text { management }=83\end{array}$ & \multirow{4}{*}{$\begin{array}{l}\text { The club has smart } \\
\text { phone applications } \\
\text { helping beneficiaries } \\
\text { to communicate } \\
\text { permanently with } \\
\text { the club }\end{array}$} & \multirow{4}{*}{55} \\
\hline & $\begin{array}{c}\text { to a certain } \\
\text { degree }\end{array}$ & 1.94 & 64.58 & 7 & 3 & 6 & $\begin{array}{c}\text { Middle } \\
\text { management }=16\end{array}$ & & \\
\hline & $\begin{array}{c}\text { to a certain } \\
\text { degree }\end{array}$ & 2.25 & 74.91 & 22 & 23 & 44 & $\begin{array}{c}\text { Executive } \\
\text { management }=89\end{array}$ & & \\
\hline 40.89 & $\begin{array}{c}\text { to a certain } \\
\text { degree }\end{array}$ & 2.33 & 77.66 & 42 & 42 & 104 & $\mathrm{All}=188$ & & \\
\hline \multirow{3}{*}{5.42} & agreeing & 2.73 & 91.16 & 8 & 6 & 69 & $\begin{array}{c}\text { Supreme } \\
\text { management }=83\end{array}$ & \multirow{4}{*}{$\begin{array}{l}\text { The club uses social } \\
\text { media to advertise } \\
\text { services provided to } \\
\text { beneficiaries }\end{array}$} & \multirow{4}{*}{56} \\
\hline & $\begin{array}{c}\text { to a certain } \\
\text { degree }\end{array}$ & 2.31 & 77.08 & 5 & 1 & 10 & $\begin{array}{c}\text { Middle } \\
\text { management }=16\end{array}$ & & \\
\hline & agreeing & 2.63 & 87.64 & 13 & 7 & 69 & $\begin{array}{c}\text { Executive } \\
\text { management }=89\end{array}$ & & \\
\hline 175.45 & agreeing & 2.65 & 88.30 & 26 & 14 & 148 & All $=188$ & & \\
\hline \multirow{3}{*}{$10.35 *$} & agreeing & 2.69 & 89.56 & 10 & 6 & 67 & $\begin{array}{c}\text { Supreme } \\
\text { management }=83\end{array}$ & \multirow{4}{*}{$\begin{array}{l}\text { The club studies the } \\
\text { beneficiaries e. } \\
\text { comments to know } \\
\text { the offered services } \\
\text { advantages and } \\
\text { disadvantages }\end{array}$} & \multirow{4}{*}{57} \\
\hline & $\begin{array}{c}\text { to a certain } \\
\text { degree }\end{array}$ & 2.19 & 82.92 & 6 & 1 & 9 & $\begin{array}{c}\text { Middle } \\
\text { management }=16\end{array}$ & & \\
\hline & agreeing & 2.46 & 82.02 & 17 & 14 & 58 & $\begin{array}{c}\text { Executive } \\
\text { management }=89\end{array}$ & & \\
\hline 122.95 & agreeing & 2.54 & 84.57 & 33 & 21 & 134 & $A l l=188$ & & \\
\hline \multirow{3}{*}{8.09} & agreeing & 2.72 & 90.76 & 8 & 7 & 68 & $\begin{array}{c}\text { Supreme } \\
\text { management }=83\end{array}$ & \multirow{4}{*}{$\begin{array}{c}\text { Answering e. } \\
\text { comments may give } \\
\text { beneficiaries good } \\
\text { impression }\end{array}$} & \multirow{4}{*}{58} \\
\hline & $\begin{array}{c}\text { to a certain } \\
\text { degree }\end{array}$ & 2.25 & 75.00 & 5 & 2 & 9 & $\begin{array}{c}\text { Middle } \\
\text { management }=16\end{array}$ & & \\
\hline & agreeing & 2.55 & 85.02 & 13 & 14 & 62 & $\begin{array}{c}\text { Executive } \\
\text { management }=89\end{array}$ & & \\
\hline 139.54 & agreeing & 2.60 & 86.70 & 26 & 23 & 139 & All $=188$ & & \\
\hline \multirow{3}{*}{5.32} & agreeing & 2.48 & 82.73 & 15 & 13 & 55 & $\begin{array}{c}\text { Supreme } \\
\text { management }=83\end{array}$ & \multirow{4}{*}{$\begin{array}{l}\text { The club allows } \\
\text { using sports } \\
\text { facilities such as } \\
\text { halls and basins and } \\
\text { leasing the same via } \\
\text { smart applications }\end{array}$} & \multirow{4}{*}{59} \\
\hline & $\begin{array}{c}\text { to a certain } \\
\text { degree }\end{array}$ & 2.00 & 66.67 & 6 & 4 & 6 & $\begin{array}{c}\text { Middle } \\
\text { management }=16\end{array}$ & & \\
\hline & agreeing & 2.46 & 82.02 & 16 & 16 & 57 & $\begin{array}{c}\text { Executive } \\
\text { management }=89\end{array}$ & & \\
\hline 73.41 & agreeing & 2.43 & 81.03 & 37 & 33 & 118 & All $=188$ & & \\
\hline \multirow{2}{*}{$13.06 *$} & agreeing & 2.64 & 87.95 & 8 & 14 & 61 & $\begin{array}{c}\text { Supreme } \\
\text { management }=83\end{array}$ & \multirow{2}{*}{$\begin{array}{c}\text { The club enhances } \\
\text { its promotional } \\
\text { campaigns via face } \\
\text { book through }\end{array}$} & \multirow{2}{*}{60} \\
\hline & $\begin{array}{c}\text { to a certain } \\
\text { degree }\end{array}$ & 2.06 & 68.75 & 7 & 1 & 8 & $\begin{array}{c}\text { Middle } \\
\text { management }=16 \\
\end{array}$ & & \\
\hline
\end{tabular}




\begin{tabular}{|c|c|c|c|c|c|c|c|c|c|}
\hline $\begin{array}{l}\text { Chi } \\
\text { square }\end{array}$ & Attitude & Average & $\begin{array}{c}\text { Out } \\
\text { weighted } \\
\text { percentage }\end{array}$ & Disagreeing & $\begin{array}{c}\text { To a } \\
\text { certain } \\
\text { degree }\end{array}$ & Agreeing & Departments & Statement content & $\mathbf{S}$ \\
\hline & agreeing & 2.58 & 86.14 & 12 & 13 & 64 & $\begin{array}{c}\text { Executive } \\
\text { management= 89 }\end{array}$ & $\begin{array}{c}\text { supporting by } \\
\text { pictures and videos }\end{array}$ & \\
\hline 118.41 & agreeing & 2.56 & 85.46 & 27 & 28 & 133 & All $=188$ & & \\
\hline \multirow{3}{*}{8.85} & agreeing & 2.53 & 84.34 & 12 & 15 & 56 & $\begin{array}{c}\text { Supreme } \\
\text { management }=83\end{array}$ & \multirow{4}{*}{$\begin{array}{c}\text { The club has a } \\
\text { system of sending } \\
\text { SMS on smart } \\
\text { phones }\end{array}$} & \multirow{4}{*}{61} \\
\hline & $\begin{array}{l}\text { to a certain } \\
\text { degree }\end{array}$ & 2.00 & 66.67 & 7 & 2 & 7 & $\begin{array}{c}\text { Middle } \\
\text { management }=16\end{array}$ & & \\
\hline & agreeing & 2.53 & 84.27 & 13 & 16 & 60 & $\begin{array}{c}\text { Executive } \\
\text { management }=89\end{array}$ & & \\
\hline 87.14 & agreeing & 2.48 & 82.80 & 32 & 33 & 123 & All $=188$ & & \\
\hline
\end{tabular}

From table (17) the research sample opinions agree upon statements numbers $(55,56,58,59$, $61)$ as chi square ranged between $(5.32: 8.85)$ with an outweighed percentage $(77.66: 88.30)$ Study of Moustafa Kawal (2018) (39) indicates the importance of using the phone application in attracting clients as they enable the organization to increase its works and reduce commercial costs.

Foad Bougnana (2008) (16) mentions the importance of using email and SMS.

Also table proves the research group's opinion disagreement on the statements number (57, 60) as chi square reached $\left(10.35^{*}\right),\left(13.06^{*}\right)$ with an outweighed percentage $(84.75),(85.46)$ respectively.

Results of study of Mariam Nariman (2012) (26) prove that social media marketing is very important for consumer in pre purchase stage as many service information are available.

The results of the study of Mariam Nariman Nomar (2012) (26) indicate that marketing through social networking sites such as Facebook is of great importance to the consumer in the pre-purchase stage, where there is a lot of information available about services, their characteristics, features and prices.

The researcher believes that social networking sites have become a means accessible to various groups of society, as these sites have multiplied and are distinguished by their availability and permanent adhesion to the beneficiaries, and they are of great importance for sports clubs because of their effective role in appealing to the beneficiaries of the services provided by sports clubs.

Table (18) Second Factor Results: Digital Transformation Requirements to Achieve the Best Competitive Advantage at Clubs

(Second Dimension: E. Marketing)

(D- E. Newspapers and digital transmission stages)

\begin{tabular}{|c|c|c|c|c|c|c|c|c|c|}
\hline $\begin{array}{l}\text { Chi } \\
\text { square }\end{array}$ & Attitude & Average & $\begin{array}{c}\text { Out } \\
\text { weighted } \\
\text { percentage }\end{array}$ & Disagreeing & $\begin{array}{c}\text { To a } \\
\text { certain } \\
\text { degree }\end{array}$ & Agreeing & Departments & Statement content & $\mathbf{S}$ \\
\hline \multirow{3}{*}{7.14} & agreeing & 2.60 & 86.75 & 10 & 13 & 60 & $\begin{array}{c}\text { Supreme } \\
\text { management= } 83\end{array}$ & \multirow{4}{*}{$\begin{array}{l}\text { The club has a you tube } \\
\text { channel to transmit } \\
\text { pictures and videos } \\
\text { about the club's } \\
\text { activities }\end{array}$} & \multirow{4}{*}{6} \\
\hline & $\begin{array}{c}\text { to a certain } \\
\text { degree }\end{array}$ & 2.13 & 70.83 & 6 & 2 & 8 & $\begin{array}{c}\text { Middle } \\
\text { management }=16\end{array}$ & & \\
\hline & agreeing & 2.57 & 85.77 & 12 & 14 & 63 & $\begin{array}{c}\text { Executive } \\
\text { management= } 89\end{array}$ & & \\
\hline 111.78 & agreeing & 2.55 & 84.93 & 28 & 29 & 131 & All $=188$ & & \\
\hline 8.51 & agreeing & 2.52 & 83.94 & 13 & 14 & 56 & $\begin{array}{c}\text { Supreme } \\
\text { management }=83\end{array}$ & The club contracts with & 63 \\
\hline
\end{tabular}




\begin{tabular}{|c|c|c|c|c|c|c|c|c|c|}
\hline $\begin{array}{l}\text { Chi } \\
\text { square }\end{array}$ & Attitude & Average & $\begin{array}{c}\text { Out } \\
\text { weighted } \\
\text { percentage }\end{array}$ & Disagreeing & $\begin{array}{c}\text { To a } \\
\text { certain } \\
\text { degree }\end{array}$ & Agreeing & Departments & Statement content & $\mathbf{S}$ \\
\hline & $\begin{array}{c}\text { to a certain } \\
\text { degree }\end{array}$ & 1.88 & 62.50 & 7 & 4 & 5 & $\begin{array}{c}\text { Middle } \\
\text { management }=16\end{array}$ & \multirow{3}{*}{$\begin{array}{c}\text { e. newspapers and } \\
\text { magazines as a method } \\
\text { of propaganda and } \\
\text { advertisement }\end{array}$} & \\
\hline & agreeing & 2.39 & 79.78 & 19 & 16 & 54 & $\begin{array}{c}\text { Executive } \\
\text { management }=89\end{array}$ & & \\
\hline 65.76 & agreeing & 2.40 & 80.14 & 39 & 34 & 115 & All $=188$ & & \\
\hline \multirow{3}{*}{$17.97 *$} & agreeing & 2.61 & 87.15 & 10 & 12 & 61 & $\begin{array}{c}\text { Supreme } \\
\text { management }=83\end{array}$ & \multirow{4}{*}{$\begin{array}{l}\text { The club contracts with } \\
\text { digital transmission } \\
\text { stages to display events } \\
\text { and matches against } \\
\text { financial return }\end{array}$} & \multirow{4}{*}{64} \\
\hline & $\begin{array}{c}\text { to a certain } \\
\text { degree }\end{array}$ & 1.81 & 60.42 & 7 & 5 & 4 & $\begin{array}{c}\text { Middle } \\
\text { management }=16\end{array}$ & & \\
\hline & agreeing & 2.45 & 81.65 & 29 & 18 & 51 & $\begin{array}{c}\text { Executive } \\
\text { management }=89\end{array}$ & & \\
\hline 62.04 & agreeing & 2.47 & 82.27 & 46 & 35 & 116 & All $=188$ & & \\
\hline \multirow{3}{*}{$17.78 *$} & agreeing & 2.63 & 87.55 & 9 & 13 & 61 & $\begin{array}{c}\text { Supreme } \\
\text { management }=83\end{array}$ & \multirow{4}{*}{$\begin{array}{l}\text { The club shall compile } \\
\text { special conditions with } \\
\text { digital transmission } \\
\text { stages when covering } \\
\text { and transmitting } \\
\text { matches and } \\
\text { championships }\end{array}$} & \multirow{4}{*}{65} \\
\hline & $\begin{array}{c}\text { to a certain } \\
\text { degree }\end{array}$ & 1.75 & 58.33 & 8 & 4 & 4 & $\begin{array}{c}\text { Middle } \\
\text { management }=16\end{array}$ & & \\
\hline & agreeing & 2.38 & 79.40 & 18 & 19 & 52 & $\begin{array}{c}\text { Executive } \\
\text { management }=89\end{array}$ & & \\
\hline 70.67 & agreeing & 2.44 & 81.21 & 35 & 36 & 117 & All $=188$ & & \\
\hline
\end{tabular}

From table (18), it is clear that the research sample agree upon statements number $(62,63)$ as chi square reached (7.14), (8.51) with outweighed percentage (84.93) (80.14) respectively.

Study of Bosaina Ghadiri (2015) (11) indicates the effect of e advertising on beneficiaries under the increasing number of internet users with focusing on advertisement content and credibility.

Also the research sample opinions disagreed on statements numbers $(64,65)$ as chi square reached $(17.97 *),(17.78 *)$ with outweighed percentage $(82.27),(81.21)$

The united company of advertising services established watch at forum which declared reaching the exclusive digital rights at Egyptian series for 4 years, which enables the forum users to enjoy all goals, matches and summaries (70).

The researcher believes that sports club administrations need to pay attention to enhancing their financial returns by attracting digital broadcasting platforms towards obtaining the rights to broadcast their sporting events, especially in light of the decline of traditional broadcasting channels.

\section{Conclusions:}

- The supreme management supports digital transformation via looking into new techniques by documenting services and trying to transform paper forms to electronic forms.

- Employees are neither trained nor provide with skills needed for attending technology permanently.

- The club's organizational structure agrees with digital transformation application requirements.

- Lack of specialized committee or unit for applying digital transformation as planned.

- Employees have no advanced digital skills such as networks management, ability to create in using digital techniques, developing digital content and computerized programming.

- Weak protection programs related to anti hacking 
- The club profits from open governmental data in developing the club's performance data.

- Artificial intelligence is, to a certain degree, at clubs to develop the athletes' level.

- Ecommerce is weakly used in facilitating commercial deals.

- The club has a web site in English and Arabic and the club's page has a service and activities description.

- Law financial appropriations allocated for research and developing website.

\section{Recommendations:}

- Finishing the national project of digital transformation considered as an important tool of achieving permanent development, developing governmental work development, providing e services and applying digital economy.

- Developing legislative frames supporting digital transformation and trying to make Egypt distinctive on great data centers manufacturing chart to be a territorial centre for data centers and information banks.

- Compiling an integrated strategy to develop human resources in conformity with achieving permanent and integrated community development in conformity with the aimed digital transformation to attend international changes and new updates.

- Clubs should adopt a clear strategy towards a digital transformation and preparing a time table for applying transformation to guarantee execution in conformity with Egypt view 2030.

- Respond to beneficiaries via developing participation channels, and activating beneficiaries, participation in developing and improving the club's decisions, policies and services.

\section{Bibliography}

\section{First: Arabic Bibliography}

1 Abdelrahman Elour (2018)

2
Abdelsabour Abdelkawy (2010)

3 Abla Zaian Bouzian (2016)

4 Ahlam Elfekky (2014)
Human resources impact, routine operations and their effect on work future, federal authority of governmental human resources, issue 8 , United Arab Emirates

Elmasry

Ecommerce and Law , Dar Al Elom Publishers, Cairo

The effect of using some technologies in developing decision making level for handball

: referees, unpublished master thesis, institute of physical and sports science and techniques, al gibali bonama university, Algergria

Information and Communication Technology and its Role in Developing Sports Management, an : Unpublished Master Thesis, Faculty of Post Graduate Studies for Physical Education, Sudan University for Science and Technology. 
5
Ahmed Adam Ahmed Mohamed (2014)

6 Ahmed Mahgoub Mousa (2015)

7 Ahmed Mohamed Ghounim (2004)

8 Ala Abdelmonim Abdalla (2014)

9 Ashour Abdelkerim (2010)

10 Beshir Arnous (2007)

11 Bosaina Ghadiri (2015)

12

13

Doaa Elhosban, Weam Elhayek
(2017)

14 Faleh Abbas Lotfy (2019)

15 Fatma Elsabiy (2019)
Information Technology Role in Improving Sports Management Performance in Some Governmental and Nongovernmental Organizations a Thesis Published on Magazine of Faculty of Physical Education, January issue , Sudan University for Science and Technology.

The Effect of Computerization on Achieving E: government Purposes, Faculty of Post Graduate Studies, Al Nilin University, Sudan.

E-management, Fields of Present and Expectation of Future , the Modern Library, Saudi Arabia

Creating and developing applications to avail the same as a cloud service, faculty of commuter science and information techniques, Al Nilin University, Sudan

The role e. Management in rationalizing public service in the US and Algeria, un published master thesis , faculty of law and political science, Mantori University, Algeria

: Artificial Intelligence, Alsahab Publishers, Cairo

E. Advertisement in Directing the Consumer's Behavior, Faculty of Economic Science, - Commercial Science and Facilitation Science, Al Arabi Ben Mehidi University, Algeria

The Role of e. Management in Achieving the Competitive Advantage at Clubs, a thesis : published on Al Elmia Magazine of Physical Education, Faculty of Physical Education for Girls, Alexandria University, volume 5 issue 5.

Challenges and Opportunities Affecting E. Government Success in Jordon, a thesis published : on science, Engineering and Information Technology Magazine, first volume, issue 2, National Research Center, Palestine.

The effect of artificial intelligence on auditing quality of accounting auditing offices in Jordon , : unpublished master thesis, faculty of scientific research deanship and post graduate studies , Girsh university, Jordon

Studying strategies of attitudes of applying blue : kitchen technique in Gulf states, al Bahrain center for strategic and international studies , Bahrain 
16 Foad Abougnana (2008)

17 Haitham Fayez Mahmoud (2016)

Hanin Abdelsalam Abou Oud,

18 Aseel Ahmed Eldarat, Aly

Mohamed Abdelshahid (2019)

19 Haroun Abdalla Eissa (2009)

21 Hend Mohamed Hamed (2010)

22 Ihab Khalifa (2018)

23
International Telecommunication Union (2018)

24
Kholoud Bent Salem Bin Saleh (2019)

25
Evaluating marketing communication fact in service organizations, unpublished master thesis, faculty of facilitation sciences, Warkala university, Algeria

E. Sports Marketing Strategy at Sports Clubs in : Egypt. unpublished doctorate thesis, Faculty of Physical Education, Alexandria University

Intelligence Things Internet in the Field of Health

: Care, issue 15, Academic Research Magazine

Designing a System for Human Development in States in the Frame of the E. Government's Project by Using Open Resources, unpublished doctorate thesis, Faculty of Computer Science and Information Technology, Al Nilin University, Sudan

Behaviors of Supplicating Information Related to E. Information Bases for Teaching Staff Members, : Islamic University of Imam Mohamed Ben Saoud, thesis published on national magazine of King Fahd, volume 20 issue 1

Ecommerce in Touristic Field, Faculty of Tourism and Hotels, Helwan University Cairo

The Blue Kitchen, the Next Technological Revolution in Finance and Administration, Future for Research and Advanced Studies, issue 3 March 2018, Abo Dhabi, Emirates

Creating Abilities in a Changeable Environment for Information and Communication Technology, Communication Development Library, Geneva , Switzerland

Using Open Governmental Data by Researchers at Faculties of Human Sciences, Sultan Kabos University, unpublished master thesis, Faculty of Arts and Social Science, Sultan Kabos, Oman

Business Establishment and Digital Transformation, a thesis published on information : Egyptian magazine, Egyptian Association for Information System and Accounting Technology, issue (21) 
26 Mariam Nariman Noumar (2012)

27 Mohamed Fathy Abdelhady (2008) :

28 Mohamed Ramadan Zaho (2006)

29 Moustafa Kawal (2018)

30 Nawal Bent Aly abdalla (2019)

31 Negm Aboud Negm (2004)

32 Omar Khalaf Salem Elsaleh (2019)

33 Omar Mohamed Saleh Aly (2018)

34 Ramadan Aly Elsayed Elmarouf (2011) Saad Ahmed Shalaby, Abdellatif
Boukhary (2008)

36 Safaa Soliman Khalil (2019)
Using Social Media and their Effects on Social Relationships "Studying a Sample of Facebook : Users in Algeria" unpublished master thesis, Faculty of Media and Communication Science, Al Haj Al Akhdar University, Algeria

Knowledge and Libraries Age, first print, High Institute of Culture, Cairo

Marketing and Advertising Researches from the Perspective of Acts Globalization and Data Electronic, Faculty of Commerce, Banha University, Egypt

Suggesting Methods of Sports Promotion to Achieve Self Finance for Algerian Clubs Professionalizing Football, unpublished doctorate thesis, Mohamed Bou Diaf University, Algeria

Digital Transformation in Oman Sultanate, : unpublished master thesis, Faculty of Arts and Social Science, Sultan Kabos University, Oman

E. Management "Strategies, Functions and : Problems", Dar ALmarikh, Saudi Arabia

Factors affecting adopting ecommerce in pharmaceutical companies in Jordon, unpublished : master thesis, faculty of economy and administrative science, Al Albit University , Jordon

Developing an application by using things internet techniques : by applying to care services , unpublished master thesis, faculty of post graduate studies, Al Nilin University, Sudan

E. Commerce in Japan and How Far Egypt Could Profit From, Jazirat Alward Library, Cairo

Electronic administration and marketing at sports clubs joining the German series of football 2007 : 2008 , analytical study, a thesis published on the first international conference magazine for physical and health education

Developing electronic office systems by using cloud computerizing techniques applying : correspondence system, unpublished doctorate thesis, faculty of computer science and information techniques, Al Nilin University, Sudan 
37

Safat Salama, Khalil Abou Koura (2014)

38 Salma Kounda (2018)

39 Srour Aly Srour (2005

40 Taweel Osama (2017)

United Nation Organization for

41 Education, Science and Culture : (2018)

42 Youssef Ahmed Abou Fara (2007)

43 Zahaf Mohamed (2018)

\section{Second: Foreign Bibliography}

- Robots age challenges and ethics, emirates center

of strategic studies and research, Abo Dhabi

Media and communication technology in the field of school management, a thesis published on

: sports creation magazine, Mohamed Amin Dabaghin university, volume 9, issue 2, Algeria

Artificial intelligence, smart systems manual, al marikh publishing house, Riyadh

Electronic management and restrictions of applying to sports management, a field study at : youth and sports directorate at al masila state, unpublished master thesis, physical activities science and techniques, Mohamed bo deif

Skills for Connected World, conceptual : memorandum, the Week of Learning by Mobile Appliances, UNESCO

E. Marketing Strategies, a behavioral approach, Al Mostakbal Publishing, Oman

E. Marketing as an approach to developing marketing information systems at Algeria sports : organizations. A thesis published on sports science magazine, volume 8 , issue 24 , Masila university, Algeria
\& Alison Davis, Matthew Merle (2019)
C. Le : :

Blockchain Competitive Advantage: Whether you are an entrepreneur, investor, or established company, learn how to win the battle for blockchain competitive advantage, Fifth Era Media

¿ $\quad$ Bruce Sinclair (2017): IoT Inc

: How Your Company Can Use the Internet of Things to Win in the Outcome Economy, McGraw-Hill Education

\& $\begin{aligned} & \text { David Furlonger, Christophe } \\ & \text { Uzureau (2019) }\end{aligned}$

¿ George Westerman (2014)

$\varepsilon \wedge$
Giacomo Veneri and Capasso (2018)

The Real Business of Blockchain: How Leaders Can

: Create Value in a New Digital Age, Harvard Business Review Press

: Leading Digital: Turning Technology into Business

Transformation, Harvard Business Review Press

Hands-On Industrial Internet of Things: Create a

: powerful Industrial loT infrastructure using Industry 4.0, Packt Publishing 
¿ Gil Gildner, Anya Gildner (2019) :
๑. Jared Tate, Andrew Knapp (2019): :

Becoming a Digital Marketer: Gaining the Hard \& Soft Skills for a Tech-Driven Marketing Career, Baltika Press

Blockchain 2035: The Digital DNA of Internet 3.0,
BlueShed LLC

E-Commerce Business Model 2020: This Book

O) Jim Work George Brand (2020)

or Joel Gurin (2014)

or Julian Singh (2017)

Kevin L. Jackson and Scott
Goessling (2018):

๑o Mohit Sharma (2018)

or $\begin{aligned} & \text { Ovidiu Vermesan, Peter Friess } \\ & \text { (2013) }\end{aligned}$

ov Rob Kitchin (2014)

$\bullet \wedge \quad$ Sarah Grand-Clement (2017)

๑q Thomas M. Siebel (2019)
: Includes: Online Marketing Strategies, Dropshipping, Amazon FBA - Step-by-Step Guide with Latest Techniques to Make Money Online and Reach Financial Freedom, Independently published Open Data Now: The Secret to Hot Startups, Smart

: Investing, Savvy Marketing, and Fast Innovation, McGraw-Hill Education

: : Open Data 101: The latest trends, challenges and research in government open data, Cooee Press
: Architecting Cloud Computing Solutions: Build cloud strategies that align technology and economics while effectively managing risk, Packt Publishing How RPA will impact the future workplace for : Governments across the world and the economy, Published by the Federal Authority for Government Human Resources, UA E

Internet of Things: Converging Technologies for Smart

: Environments and Integrated Ecosystems, River Publishers

The Data Revolution: Big Data, Open Data, Data

: Infrastructures and Their Consequences, SAGE Publications Ltd

: development for a connected world, RAND Europe Digital technology's role in enabling skills Mass Extinction, Rosetta Books
: Digital Transformation: Survive and Thrive in an Era of

\section{Third: International Information Network}

60

https://www.sis.gov.eg/Story/178669/
: Website of Information Authority - 15 November 2018: The partnership between the government and private sector is a basis of permanent development objectives 
61

http://www.mcit.gov.eg/Ar/Media_Center/P ress_Room/Press_Releases/41556

62

https://www.youm7.com/story/2019/11/22

63 https://www.elwatannews.com/news/details/ $\underline{4705703}$

64 https://arabic.sport360.com/article/

65 https://www.bneconomy.com/9274

66 https://www.elwatannews.com/news/details/ 1273513

67 https://www.rosaelyoussef.com/519910

68

http://www.mcit.gov.eg/Ar/Media_Center/P ress_Room/Press_Releases/41556

69

https://www.alayam.com/Article/sportarticle/414326/

70

https://www.youm7.com/story/2019/5/27/
: Website of Ministry of Communication and Information Technology - Cairo 13 January 2020: Officially , Ministry of Planning Provides Ministry of Communication with Digital Transformation Projects

: website of Al Youm Al Sabei Newspaper 22 November 2019: for the first time Egypt operate a communication satellite to apply digital transformation

: Website of Alwatan Newspaper 18 April 2020: Sobhy discusses with Microsoft : digital transformation of projects of " youth and sports"

: Website of sport 360 - 07 November 2019: Al Ahly Club uncover strategy of digital transformation for all members' services

: Site of baladna news economic 03 August 2019: Heliopolis club and Kuwait national bank sign a cooperation protocol of digital transformation of the club in cooperation with Raya systems

: Alwatan newspaper 23 July 2016: Egypt telecom signing a protocol of cooperation with "Smoha Club"

: Website of Ros Al Yossef newspaper 30 December 2019: Dr./ Ahmed Farouk Abdelkader writes: Digital Transformation in Egyptian Sports

: Website of Ministry of Communication and Information Technology 13 January 2020: Officially, Ministry of Planning Provides Ministry of Communication with Digital Transformation Projects

: Prof. Dr. / Faisal Elmala, website of alyam newspaper sports field and artificial intelligence

: Alyom alsabei 27/05/2019, exclusively, the Egyptian serious digitally on Watch iT 